This paper is dedicated to Robert Tijdeman on the occasion of his 75 th birthday.

\title{
SOME CONSTRUCTIONS FOR THE HIGHER-DIMENSIONAL THREE-DISTANCE THEOREM
}

\author{
VALÉRIE BERTHÉ AND DONG HAN KIM
}

\begin{abstract}
For a given real number $\alpha$, let us place the fractional parts of the points $0, \alpha, 2 \alpha, \cdots,(N-1) \alpha$ on the unit circle. These points partition the unit circle into intervals having at most three lengths, one being the sum of the other two. This is the three distance theorem. We consider a two-dimensional version of the three distance theorem obtained by placing on the unit circle the points $n \alpha+m \beta$, for $0 \leq$ $n, m<N$. We provide examples of pairs of real numbers $(\alpha, \beta)$, with $1, \alpha, \beta$ rationally independent, for which there are finitely many lengths between successive points (and in fact, seven lengths), with $(\alpha, \beta)$ not badly approximable, as well as examples for which there are infinitely many lengths.
\end{abstract}

\section{INTRODUCTION}

For a given real number $\alpha$ in $(0,1)$, let us place the points $\{0\},\{\alpha\}$, $\{2 \alpha\}, \cdots,\{(N-1) \alpha\}$ on the unit circle, where $\{x\}$ denotes as usual the fractional part of $x$. These points partition the unit circle into $N$ intervals having at most three lengths, one being the sum of the other two. This property is known as the three distance theorem and can be seen as a geometric interpretation of good approximation properties of the Farey partial convergents in the continued fraction expansion of $\alpha$. In the literature, this theorem is called the Steinhaus theorem, the three length, the three gap, or else, the three step theorem.

The three distance theorem was initially conjectured by Steinhaus, first proved V. T. Sós [S58] and Surányi [Suŕ58], and then by Slater Sla64, Świerczkowski [Ś59], Halton [Hal65]. A survey of the different approaches used by these authors is to be found for instance in AB98, vR88, Sla67, Lan91]. More recent proofs have also been given in [vR88, Lan91, or in

2010 Mathematics Subject Classification. Primary 11J13; Secondary 11J70, 11J71, 11B75, 11A55.

Key words and phrases. Gap theorems, continued fractions, distribution modulo 1.

This work was supported by the Agence Nationale de la Recherche through the project DynA3S (ANR-13-BS02-0003), National Research Foundation of Korea (NRF2015R1A2A2A01007090) and by the Fondation Sciences Mathématiques de Paris (FSMP) through the support for the visit of Dong Han Kim at IRIF in 2016. 
[MS17] relying on the properties of space of two-dimensional Euclidean lattices. See also [Ble91, PSZ16] for the study of the limiting distribution of the gaps.

There exist numerous generalizations of the three gap theorem. Let us quote for instance generalizations for groups [FS92], for some isometries of compact Riemannian manifolds [BS08], or else for interval exchange transformations [Dia17]. Among generalizations, there are two natural Diophantine frameworks that are dual, namely distance theorems for toral translations on the $d$-dimensional torus $\mathbb{T}^{d}$ (see e.g. [Che07, Che14, Vij08]), and distance theorems for linear forms in $d$ variables on the one-dimensional torus $\mathbb{T}$. This is the framework of the present paper, where we focus on linear forms in two variables, and consider points $m \alpha+n \beta$, for $0 \leq n, m<N$, in $\mathbb{T}$.

This generalization has been considered by Erdös (as recalled in GS93]) and also in [Lia79, CG76, GS93, FH95, Che00, BHJ ${ }^{+}$12, HM17]. See also CGVZ02 for the number of so-called primitive gaps. In particular, the following is proved in Che00]. Let $\alpha_{1}, \ldots, \alpha_{d} \in \mathbb{T}(d \geq 3)$ and $2 \leq n_{1} \leq$ $\ldots \leq n_{d}$ be integers. The set $\left\{\sum_{i=1}^{d} k_{i} \alpha_{i}, 0 \leq k_{i}<n_{i}, i=1, \ldots, d\right\}$ divides $\mathbb{T}$ into intervals whose lengths take at most $\prod_{i=1}^{d-1} n_{i}+3 \prod_{i=1}^{d-2} n_{i}+1$ values. When $d=2$, the upper bound is $N+3$ for the case of interest here $(m \alpha+n \beta$, for $0 \leq n, m<N)$, as proved in [GS93].

There are natural cases where it is known that the number of distances is bounded (with respect to $N$, for the points $m \alpha+n \beta$, for $0 \leq n, m<N$ ). This is the case if $1, \alpha, \beta$ are rationally dependent (this has been proved by Holzman, as recalled in [GS93]). Badly approximable vectors $(\alpha, \beta)$ have also been proved by Boshernitzan and Dyson to produce a finite number of distances. For a proof, see $\left[\mathrm{BHJ}^{+} 12\right]$. Nevertheless, it is proved in [HM17] that the number of lengths is generically unbounded, with an approach via homogeneous dynamics based on the ergodic properties of the diagonal action on the space of lattices. However, no explicit examples of this generic situation were known. The object of the present paper is to construct such examples.

Our main result is the following.

Theorem 1.1. Consider the set $E_{N}(\alpha, \beta):=\{n \alpha+m \beta \in \mathbb{T}: 0 \leq n, m<$ $N\}$, and let $\Delta\left(E_{N}(\alpha, \beta)\right)$ stand for the set of distances between neighbor points of $E_{N}(\alpha, \beta)$. We provide effective constructions for the following existence results. 
(i) There exist $(\alpha, \beta)$, with $1, \alpha, \beta$ rationally independent and $(\alpha, \beta)$ not badly approximable, such that:

$$
\forall N, \# \Delta\left(E_{N}(\alpha, \beta)\right) \leq 7 .
$$

(ii) There exist $(\alpha, \beta)$, with $1, \alpha, \beta$ rationally independent, such that:

$$
\limsup _{N \rightarrow \infty} \# \Delta\left(E_{N}(\alpha, \beta)\right)=\infty .
$$

Our proof avoids the use of a higher-dimensional analogue of continued fractions. We rely on the (regular) continued fraction expansions of $\alpha$ and $\beta$ and we combine several 'rectangular' levels of points of the form $n \alpha+m \beta$, for $0 \leq n<N$ and $0 \leq m<M$, where $N$ or $M$ is a denominator of a principal convergent of $\alpha$ or $\beta$.

We ask the question of the minimality of the number of lengths: is it possible to find $(\alpha, \beta)$, with $1, \alpha, \beta$ rationally independent, such that $\# \Delta\left(E_{N}(\alpha, \beta)\right) \leq 6$, for all $N$ ?

As an application and motivation for this theorem, one deduces results on frequencies of square factors in two-dimensional Sturmian words, such as studied in [BV00, BT02]. Two-dimensional Sturmian words are defined as codings of $\mathbb{Z}^{2}$-actions by rotations on the one-dimensional torus $\mathbb{T}$. More precisely, let $\alpha, \beta, \rho$ be real numbers, with $1, \alpha, \beta$ rationally independent, and $0<\alpha+\beta<1$. A two-dimensional Sturmian word over the threeletter alphabet $\{1,2,3\}$ (with parameters $\alpha, \beta, \rho)$ is defined as a function $f: \mathbb{Z}^{2} \rightarrow\{1,2,3\}$, with, for all $(m, n) \in \mathbb{Z}^{2},(f(m, n)=i \Longleftrightarrow m \alpha+n \beta+\rho \in$ $I_{i}$ modulo 1), where either $I_{1}=[0, \alpha), I_{2}=[\alpha, \alpha+\beta), I_{3}=[\alpha+\beta, 1)$, or $I_{1}=(0, \alpha], I_{2}=(\alpha, \alpha+\beta], I_{3}=(\alpha+\beta, 1]$. According to [BV00], frequencies of square factors of size $N$ are equal to the lengths obtained by putting on $\mathbb{T}$ the points $-n \alpha-m \beta$, for $-1 \leq n \leq N-1,0 \leq m \leq N$. One thus has a correspondence between lengths and frequencies, whereas gap theorems correspond to return words. Note that the convergence toward frequencies (expressed in terms of balance properties) has been considered in [BT02]. More generally, for results of the same flavor for cut and project sets generalizing the Sturmian framework, see [HKWS16, HJKW17].

Contents of the paper. Let us briefly sketch the contents of this paper. Notation are introduced in Section 2 together with a basic lemma (Lemma 2.1) that allows one to express in a convenient way the clockwise neighbor of a point of the form $n \alpha+m \beta$. A construction providing pairs $(\alpha, \beta)$ with a bounded number of lengths is described in Section 3 , while the case of an unbounded number of lengths is handled in Section 4; Statement (i) of Theorem 1.1 is proved in Section 3, and Statement (ii) in Section 4. 
Acknowledgements. Our deepest gratitude goes to Robert Tijdeman who has been a constant source of inspiration for his input in word combinatorics and discrete mathematics through his deep and wide understanding of equidistribution theory. We would like to thank Alan Haynes for pointing out the problem (see $\left[\mathrm{ADG}^{+} 16\right]$ ) and for stimulating discussions. We also would like to thank Damien Jamet and Thomas Fernique for computer simulations. Lastly, we gratefully thank the referee for his valuable comments which helped to improve the manuscript.

\section{Preliminaries}

Let $\mathbb{T}=\mathbb{R} / \mathbb{Z}$. Let $\alpha, \beta$ be real numbers in $(0,1)$. We assume in all this paper that $1, \alpha, \beta$ are rationally independent.

For $q, q^{\prime}$ positive integers, we define

$$
E_{q, q^{\prime}}(\alpha, \beta):=\left\{n \alpha+m \beta \in \mathbb{T}: 0 \leq n<q, 0 \leq m<q^{\prime}\right\}
$$

and when $q=q^{\prime}$, we use the notation $E_{N}(\alpha, \beta):=E_{q, q^{\prime}}(\alpha, \beta)$, with $N:=$ $q=q^{\prime}$. We furthermore consider

$$
\mathcal{E}_{q, q^{\prime}}(\alpha, \beta):=\left\{(n, m): 0 \leq n<q, 0 \leq m<q^{\prime}\right\}
$$

We will also use the shorthand notation $E_{q, q^{\prime}}, E_{N}$ and $\mathcal{E}_{q, q^{\prime}}$.

Points in $E_{q, q^{\prime}}(\alpha, \beta)$ are considered as positioned on the unit circle oriented clockwise endowed with the origin point 0 . The point $n \alpha+m \beta$ is thus considered as positioned at distance $\{n \alpha+m \beta\}$ from the origin point 0 . The point that is located immediately after $n \alpha+m \beta$ clockwise on the unit circle, that is, its clockwise neighbor, is denoted as $\Phi_{q, q^{\prime}}(n \alpha+m \beta)$, or $\Phi(n \alpha+m \beta)$, if there is no confusion. This thus defines a map $\Phi_{q, q^{\prime}}$ on $E_{q, q^{\prime}}$ called the neighbor map. For two points $a, b$ in $\mathbb{T}$, the interval $(a, b)$ in $\mathbb{T}$ corresponds to the interval considered clockwise on the unit circle with endpoints being respectively $a$ and $b$. The set $E_{q, q^{\prime}}$ thus partitions the unit circle into disjoint intervals $\left(n \alpha+m \beta, \Phi_{q, q^{\prime}}(n \alpha+m \beta)\right)$, for $(n, m) \in \mathcal{E}_{q, q^{\prime}}$.

For a finite subset $E$ of $\mathbb{T}=\mathbb{R} / \mathbb{Z}$, we denote by $\Delta(E)$ the set of distances between neighbor points of $E$ (again with distances being counted clockwise). For any $(n, m)$ in $\mathcal{E}_{q, q^{\prime}}, \Delta_{q, q^{\prime}}(n, m)$ (or $\Delta(n, m)$ if there is no confusion) stands for the distance between $n \alpha+m \beta$ and $\Phi_{q, q^{\prime}}(n \alpha+m \beta)$.

For any positive integer $q$, we define the nonnegative integer $|n|_{q}$ as

$$
|n|_{q} \equiv n \quad(\bmod q), \quad \text { and } \quad 0 \leq|n|_{q}<q .
$$

We will consider in the following the map $n \mapsto|n+r|_{q}$, for a given integer $r$. In particular, if $0 \leq r<q$, one has $|n+r|_{q}=n+r$ if $0 \leq n<q-r$, and $|n+r|_{q}=n+r-q$ if $q-r \leq n<q$. 
Let $q, q^{\prime}$ be two given positive integers. We also assume that $q$ and $q^{\prime}$ are coprime. Then, for any integers $r, r^{\prime} \operatorname{such}$ that $\operatorname{gcd}(r, q)=1, \operatorname{gcd}\left(r^{\prime}, q^{\prime}\right)=1$, $0<|r|<q, 0<\left|r^{\prime}\right|<q^{\prime}$, the map

$$
\varphi_{q, q^{\prime}}: \mathcal{E}_{q, q^{\prime}} \rightarrow \mathcal{E}_{q, q^{\prime}}, \quad(n, m) \mapsto\left(|n+r|_{q},\left|m+r^{\prime}\right|_{q^{\prime}}\right)
$$

is a cyclic permutation of $\mathcal{E}_{q, q^{\prime}}$. We thus will be able to describe the elements of the set $\mathcal{E}_{q, q^{\prime}}$ as the elements of the orbit of $(0,0)$ under the map $\varphi_{q, q^{\prime}}$. In particular, for each $(n, m)$, with $0 \leq n<q, 0 \leq m<q^{\prime}$, there exists a unique $k$, with $0 \leq k<q q^{\prime}$, satisfying $(n, m)=\left(|k r|_{q},\left|k r^{\prime}\right|_{q^{\prime}}\right)$. Indeed, since $1, \alpha, \beta$ are rationally independent, the following map $\phi_{q, q^{\prime}}$ acting on $E_{q, q^{\prime}}(\alpha, \beta)$, and defined by

$$
\phi_{q, q^{\prime}}(n \alpha+m \beta)=\left\langle\varphi_{q, q^{\prime}}(n, m),(\alpha, \beta)\right\rangle
$$

is easily seen to be injective, and thus surjective.

Let $\left(a_{i}\right)_{i \geq 1},\left(a_{j}^{\prime}\right)_{j \geq 1}$ stand for the respective sequences of partial quotients of $\alpha$ and $\beta$ in their continued fraction expansions, and denote by $\left(q_{i}\right)_{i \geq 1}$, $\left(q_{j}^{\prime}\right)_{j \geq 1}$ the denominators of their principal convergents. Note that we will make a strong use of

$$
q_{k}\left\|q_{k-1} \alpha\right\|+q_{k-1}\left\|q_{k} \alpha\right\|=1 \text {. }
$$

Here we denote $\|t\|$ by the distance to the nearest integer of $t \in \mathbb{R}$.

We now consider $E_{q_{i}, q_{j}^{\prime}}(\alpha, \beta)=\left\{n \alpha+m \beta \in \mathbb{T}: 0 \leq n<q_{i}, 0 \leq m<q_{j}^{\prime}\right\}$, for indexes $i, j$ for which we assume that they satisfy $q_{j}^{\prime}=b^{\prime} q_{i}+1$ for some positive integer $b^{\prime}$. Note that $b^{\prime} q_{j-1}^{\prime}$ is coprime with $q_{j}^{\prime}$ since $b^{\prime}$ and $q_{j-1}^{\prime}$ are coprime with $q_{j}^{\prime}$. We take $r:=-(-1)^{i} q_{i-1}$ and $r^{\prime}:=(-1)^{j} b^{\prime} q_{j-1}^{\prime}$. We consider the following cyclic permutations acting respectively on $\mathcal{E}_{q_{i}, q_{j}^{\prime}}(\alpha, \beta)$ and $E_{q_{i}, q_{j}^{\prime}}(\alpha, \beta)$ :

$$
\begin{gathered}
\varphi_{q_{i}, q_{j}^{\prime}}: n \alpha+m \beta \mapsto\left(\left|n-(-1)^{i} q_{i-1}\right|_{q_{i}},\left|m+(-1)^{j} b^{\prime} q_{j-1}^{\prime}\right|_{q_{j}^{\prime}}\right), \\
\phi_{q_{i}, q_{j}^{\prime}}: n \alpha+m \beta \mapsto\left|n-(-1)^{i} q_{i-1}\right|_{q_{i}} \alpha+\left|m+(-1)^{j} b^{\prime} q_{j-1}^{\prime}\right|_{q_{j}^{\prime}} \beta .
\end{gathered}
$$

Lemma 2.1 below shows that, under Assumption (2.3), the clockwise neighbor point $\Phi_{q_{i}, q_{j}^{\prime}}(n \alpha+m \beta)$ of $n \alpha+m \beta$ in $E_{q_{i}, q_{j}^{\prime}}(\alpha, \beta)$ is exactly $\phi(n \alpha+m \beta)$, by using the shorthand notation $\phi=\phi_{q_{i}, q_{j}^{\prime}}$. Lemma 2.1 will be used in the proofs of both statements of Theorem 1.1. In particular, it will play a crucial role in Section 3 for the case of a bounded number of lengths. Indeed, in order to count the number of lengths for a square set of points $\mathcal{E}_{N}$, we consider several rectangular subsets of points in $\mathcal{E}_{N}$, i.e., several levels of

\footnotetext{
${ }^{1}$ This map is well-defined since $1, \alpha, \beta$ are rationally independent.
} 
points in $E_{N}$, with the points of $E_{q_{i}, q_{j}^{\prime}}$ corresponding to the first level. Further levels of points will then be inserted or removed. Note that Lemma 2.1 provides a case where there are only 4 possible lengths.

Lemma 2.1. Let $\alpha, \beta$ be real numbers in $(0,1)$ such that $1, \alpha, \beta$ are rationally independent. Let $\left(q_{i}\right)_{i \geq 1},\left(q_{j}^{\prime}\right)_{j \geq 1}$ stand for the denominators of their principal convergents. We assume that for some $i, j \geq 1$

$$
q_{j}^{\prime}=b^{\prime} q_{i}+1
$$

for some positive integer $b^{\prime}$. Let

$$
\phi(n \alpha+m \beta):= \begin{cases}\left|n+q_{i-1}\right|_{q_{i}} \alpha+\left|m-b^{\prime} q_{j-1}^{\prime}\right|_{q_{j}^{\prime}} \beta, & \text { if } i, j \text { are odd, } \\ \left|n+q_{i-1}\right|_{q_{i}} \alpha+\left|m+b^{\prime} q_{j-1}^{\prime}\right|_{q_{j}^{\prime}} \beta, & \text { if } i \text { is odd, } j \text { is even, } \\ \left|n-q_{i-1}\right|_{q_{i}} \alpha+\left|m-b^{\prime} q_{j-1}^{\prime}\right|_{q_{j}^{\prime}} \beta, & \text { if } i \text { is even, } j \text { is odd, } \\ \left|n-q_{i-1}\right|_{q_{i}} \alpha+\left|m+b^{\prime} q_{j-1}^{\prime}\right|_{q_{j}^{\prime}} \beta, & \text { if } i, j \text { are even. }\end{cases}
$$

Then $\phi$ is a permutation of $E_{q_{i}, q_{j}^{\prime}}(\alpha, \beta)$.

Under the further assumption that

$$
\left\|q_{j}^{\prime} \beta\right\|<\left\|q_{i-1} \alpha\right\|-b^{\prime}\left\|q_{j-1}^{\prime} \beta\right\|,
$$

then the maps $\Phi$ and $\phi$ coincide, that is, the point $\Phi(n \alpha+m \beta)$ that is located immediately after $n \alpha+m \beta$ clockwise on the unit circle, for $0 \leq n<q_{i}$, $0 \leq m<q_{j}^{\prime}$, is $\phi(n \alpha+m \beta)$. Moreover, the distance (counted clockwise) $\Delta(n, m)$ between $n \alpha+m \beta$ and $\phi(n \alpha+m \beta)$, for $0 \leq n<q_{i}, 0 \leq m<q_{j}^{\prime}$, takes one of the following values

$$
\begin{array}{ll}
\left\|q_{i-1} \alpha\right\|-b^{\prime}\left\|q_{j-1}^{\prime} \beta\right\|, & \left\|q_{i-1} \alpha\right\|-b^{\prime}\left\|q_{j-1}^{\prime} \beta\right\|-\left\|q_{j}^{\prime} \beta\right\|, \\
\left\|q_{i-1} \alpha\right\|-b^{\prime}\left\|q_{j-1}^{\prime} \beta\right\|+\left\|q_{i} \alpha\right\|, & \left\|q_{i-1} \alpha\right\|-b^{\prime}\left\|q_{j-1}^{\prime} \beta\right\|+\left\|q_{i} \alpha\right\|-\left\|q_{j}^{\prime} \beta\right\| .
\end{array}
$$

More precisely, if e.g. $i, j$ are odd, then $\Delta(n, m)$ equals:

$$
\begin{array}{ll}
\left\|q_{i-1} \alpha\right\|-b^{\prime}\left\|q_{j-1}^{\prime} \beta\right\|, & 0 \leq n<q_{i}-q_{i-1}, b^{\prime} q_{j-1}^{\prime} \leq m<q_{j}^{\prime}, \\
\left\|q_{i-1} \alpha\right\|-b^{\prime}\left\|q_{j-1}^{\prime} \beta\right\|-\left\|q_{j}^{\prime} \beta\right\|, & 0 \leq n<q_{i}-q_{i-1}, 0 \leq m<b^{\prime} q_{j-1}^{\prime}, \\
\left\|q_{i-1} \alpha\right\|-b^{\prime}\left\|q_{j-1}^{\prime} \beta\right\|+\left\|q_{i} \alpha\right\|, & q_{i}-q_{i-1} \leq n<q_{i}, b^{\prime} q_{j-1}^{\prime} \leq m<q_{j}^{\prime}, \\
\left\|q_{i-1} \alpha\right\|-b^{\prime}\left\|q_{j-1}^{\prime} \beta\right\|+\left\|q_{i} \alpha\right\|-\left\|q_{j}^{\prime} \beta\right\|, & q_{i}-q_{i-1} \leq n<q_{i}, 0 \leq m<b^{\prime} q_{j-1}^{\prime},
\end{array}
$$

and if e.g. $i, j$ are even, then $\Delta(n, m)$ equals:

$$
\begin{array}{ll}
\left\|q_{i-1} \alpha\right\|-b^{\prime}\left\|q_{j-1}^{\prime} \beta\right\|, & q_{i-1} \leq n<q_{i}, 0 \leq m<q_{j}^{\prime}-b^{\prime} q_{j-1}^{\prime}, \\
\left\|q_{i-1} \alpha\right\|-b^{\prime}\left\|q_{j-1}^{\prime} \beta\right\|-\left\|q_{j}^{\prime} \beta\right\|, & q_{i-1} \leq n<q_{i}, q_{j}^{\prime}-b^{\prime} q_{j-1}^{\prime} \leq m<q_{j}^{\prime}, \\
\left\|q_{i-1} \alpha\right\|-b^{\prime}\left\|q_{j-1}^{\prime} \beta\right\|+\left\|q_{i} \alpha\right\|, & 0 \leq n<q_{i-1}, 0 \leq m<q_{j}^{\prime}-b^{\prime} q_{j-1}^{\prime}, \\
\left\|q_{i-1} \alpha\right\|-b^{\prime}\left\|q_{j-1}^{\prime} \beta\right\|+\left\|q_{i} \alpha\right\|-\left\|q_{j}^{\prime} \beta\right\|, & 0 \leq n<q_{i-1}, q_{j}^{\prime}-b^{\prime} q_{j-1}^{\prime} \leq m<q_{j}^{\prime} .
\end{array}
$$

Similar formulas hold for the other cases. 
Proof. Recall that $\operatorname{gcd}\left(b^{\prime} q_{j-1}^{\prime}, q_{j}^{\prime}\right)=1$.

We first assume that $i, j$ are odd. Then, $q_{i-1} \alpha-p_{i-1}=\left\|q_{i-1} \alpha\right\|, q_{j-1}^{\prime} \beta-$ $p_{j-1}^{\prime}=\left\|q_{j-1}^{\prime} \beta\right\|$ and $q_{i} \alpha-p_{i}=-\left\|q_{i} \alpha\right\|, q_{j}^{\prime} \beta-p_{j}^{\prime}=-\left\|q_{j}^{\prime} \beta\right\|$. Therefore, $q_{i-1} \alpha-b^{\prime} q_{j-1}^{\prime} \beta=\left\|q_{i-1} \alpha\right\|-b^{\prime}\left\|q_{j-1}^{\prime} \beta\right\|+\left(p_{i-1}-b^{\prime} p_{j-1}^{\prime}\right)$. It follows that

$$
\begin{aligned}
& \phi(n \alpha+m \beta)-(n \alpha+m \beta) \\
& =\left|n+q_{i-1}\right|_{q_{i}} \alpha+\left|m-b^{\prime} q_{j-1}^{\prime}\right|_{q_{j}^{\prime}} \beta-(n \alpha+m \beta) \\
& = \begin{cases}q_{i-1} \alpha-b^{\prime} q_{j-1}^{\prime} \beta, & 0 \leq n<q_{i}-q_{i-1}, b^{\prime} q_{j-1}^{\prime} \leq m<q_{j}^{\prime}, \\
q_{i-1} \alpha-\left(b^{\prime} q_{j-1}^{\prime}-q_{j}^{\prime}\right) \beta, & 0 \leq n<q_{i}-q_{i-1}, 0 \leq m<b^{\prime} q_{j-1}^{\prime}, \\
\left(q_{i-1}-q_{i}\right) \alpha-b^{\prime} q_{j-1}^{\prime} \beta, & q_{i}-q_{i-1} \leq n<q_{i}, \quad b^{\prime} q_{j-1}^{\prime} \leq m<q_{j}^{\prime}, \\
\left(q_{i-1}-q_{i}\right) \alpha-\left(b^{\prime} q_{j-1}^{\prime}-q_{j}^{\prime}\right) \beta, & q_{i}-q_{i-1} \leq n<q_{i}, 0 \leq m<b^{\prime} q_{j-1}^{\prime} .\end{cases}
\end{aligned}
$$

Let us assume that Equation (2.3) holds. Let $\widetilde{\Delta}(n, m)$ stand for the distance (counted clockwise) between $n \alpha+m \beta$ and $\phi(n \alpha+m \beta)$, for $0 \leq n<q_{i}$, $0 \leq m<q_{j}^{\prime}$. Denote $D:=\left\|q_{i-1} \alpha\right\|-b^{\prime}\left\|q_{j-1}^{\prime} \beta\right\|$. One has

$$
\widetilde{\Delta}(n, m)= \begin{cases}D, & 0 \leq n<q_{i}-q_{i-1}, b^{\prime} q_{j-1}^{\prime} \leq m<q_{j}^{\prime}, \\ D-\left\|q_{j}^{\prime} \beta\right\|, & 0 \leq n<q_{i}-q_{i-1}, 0 \leq m<b^{\prime} q_{j-1}^{\prime}, \\ D+\left\|q_{i} \alpha\right\|, & q_{i}-q_{i-1} \leq n<q_{i}, b^{\prime} q_{j-1}^{\prime} \leq m<q_{j}^{\prime}, \\ D+\left\|q_{i} \alpha\right\|-\left\|q_{j}^{\prime} \beta\right\|, & q_{i}-q_{i-1} \leq n<q_{i}, 0 \leq m<b^{\prime} q_{j-1}^{\prime} .\end{cases}
$$

By Equation (2.3), for all four cases, the values of the right hand side are positive and less than 1 .

We now assume that $i$ is odd and $j$ is even. Then, $q_{i-1} \alpha+b^{\prime} q_{j-1}^{\prime} \beta=$ $\Delta+\left(b p_{i-1}+b^{\prime} p_{j-1}^{\prime}\right)$. Similarly, we deduce that

$$
\widetilde{\Delta}(n, m)= \begin{cases}D, & 0 \leq n<q_{i}-q_{i-1}, 0 \leq m<q_{j}^{\prime}-b^{\prime} q_{j-1}^{\prime}, \\ D-\left\|q_{j}^{\prime} \beta\right\|, & 0 \leq n<q_{i}-q_{i-1}, q_{j}^{\prime}-b^{\prime} q_{j-1}^{\prime} \leq m<q_{j}^{\prime}, \\ D+\left\|q_{i} \alpha\right\|, & q_{i}-q_{i-1} \leq n<q_{i}, 0 \leq m<q_{j}^{\prime}-b^{\prime} q_{j-1}^{\prime}, \\ D+\left\|q_{i} \alpha\right\|-\left\|q_{j}^{\prime} \beta\right\|, & q_{i}-q_{i-1} \leq n<q_{i}, q_{j}^{\prime}-b^{\prime} q_{j-1}^{\prime} \leq m<q_{j}^{\prime} .\end{cases}
$$

If $i$ is even and $j$ is odd, then

$$
\widetilde{\Delta}(n, m)= \begin{cases}D, & q_{i-1} \leq n<q_{i}, b^{\prime} q_{j-1}^{\prime} \leq m<q_{j}^{\prime}, \\ D-\left\|q_{j}^{\prime} \beta\right\|, & q_{i-1} \leq n<q_{i}, 0 \leq m<b^{\prime} q_{j-1}^{\prime}, \\ D+\left\|q_{i} \alpha\right\|, & 0 \leq n<q_{i-1}, b^{\prime} q_{j-1}^{\prime} \leq m<q_{j}^{\prime}, \\ D+\left\|q_{i} \alpha\right\|-\left\|q_{j}^{\prime} \beta\right\|, & 0 \leq n<q_{i-1}, 0 \leq m<b^{\prime} q_{j-1}^{\prime} .\end{cases}
$$

Lastly, if $i, j$ are even, then

$$
\widetilde{\Delta}(n, m)= \begin{cases}D, & q_{i-1} \leq n<q_{i}, 0 \leq m<q_{j}^{\prime}-b^{\prime} q_{j-1}^{\prime}, \\ D-\left\|q_{j}^{\prime} \beta\right\|, & q_{i-1} \leq n<q_{i}, q_{j}^{\prime}-b^{\prime} q_{j-1}^{\prime} \leq m<q_{j}^{\prime}, \\ D+\left\|q_{i} \alpha\right\|, & 0 \leq n<q_{i-1}, 0 \leq m<q_{j}^{\prime}-b^{\prime} q_{j-1}^{\prime}, \\ D+\left\|q_{i} \alpha\right\|-\left\|q_{j}^{\prime} \beta\right\|, & 0 \leq n<q_{i-1}, q_{j}^{\prime}-b^{\prime} q_{j-1}^{\prime} \leq m<q_{j}^{\prime} .\end{cases}
$$


Hence, we conclude that, for the four cases obtained by considering the parity of $i, j$, one gets

$$
\begin{aligned}
& \#\{(n, m): \widetilde{\Delta}(n, m)=D\}=\left(q_{i}-q_{i-1}\right)\left(q_{j}^{\prime}-b^{\prime} q_{j-1}^{\prime}\right), \\
& \#\left\{(n, m): \widetilde{\Delta}(n, m)=D-\left\|q_{j}^{\prime} \beta\right\|\right\}=\left(q_{i}-q_{i-1}\right) b^{\prime} q_{j-1}^{\prime}, \\
& \#\left\{(n, m): \widetilde{\Delta}(n, m)=D+\left\|q_{i} \alpha\right\|\right\}=q_{i-1}\left(q_{j}^{\prime}-b^{\prime} q_{j-1}^{\prime}\right), \\
& \#\left\{(n, m): \widetilde{\Delta}(n, m)=D+\left\|q_{i} \alpha\right\|-\left\|q_{j}^{\prime} \beta\right\|\right\}=q_{i-1} b^{\prime} q_{j-1}^{\prime} .
\end{aligned}
$$

We now show the map $\phi$ sends a point to its neighbor point in the clockwise direction, that is, $\phi$ and $\Phi$ coincide. It is sufficient to notice that the $q_{i} q_{j}^{\prime}$ intervals $((n \alpha+m \beta), \phi(n \alpha+m \beta))$ of $\mathbb{T}$ never overlap. Indeed, the sum of their lengths $\widetilde{\Delta}(n, m)$ equals 1 , as shown below using (2.2):

$$
\begin{aligned}
1 & =q_{j}^{\prime}-b^{\prime} q_{i} \\
& =\left(q_{i}\left\|q_{i-1} \alpha\right\|+q_{i-1}\left\|q_{i} \alpha\right\|\right) q_{j}^{\prime}-b^{\prime}\left(q_{j}^{\prime}\left\|q_{j-1}^{\prime} \beta\right\|+q_{j-1}^{\prime}\left\|q_{j}^{\prime} \beta\right\|\right) q_{i} \\
& =q_{i} q_{j}^{\prime}\left(\left\|q_{i-1} \alpha\right\|-b^{\prime}\left\|q_{j-1}^{\prime} \beta\right\|\right)+q_{i-1} q_{j}^{\prime}\left\|q_{i} \alpha\right\|-b^{\prime} q_{j-1}^{\prime} q_{i}\left\|q_{j}^{\prime} \beta\right\| \\
& =\sum_{(m, n) \in \mathcal{E}_{q_{i}, q_{j}^{\prime}}}(\phi(n \alpha+m \beta)-(n \alpha+m \beta)) .
\end{aligned}
$$

Remark 2.2. The map $\varphi_{q_{i}, q_{j}^{\prime}}$ (associated with $\phi$ through (2.1)) is an exchange of 4 rectangles on $\mathcal{E}_{q_{i}, q_{j}^{\prime}}$. For an illustration, see Figure 1 below.

Remark 2.3. According to [CGVZ02, a distance in $\Delta\left(E_{q, q^{\prime}}(\alpha, \beta)\right)$ is said to be primitive if it is not a sum of shorter lengths (not necessarily distinct). It is proved in the same paper CGVZ02 that there are at most 4 primitive lengths for $E_{q, q^{\prime}}(\alpha, \beta)$. The lengths given in Lemma 2.1 are primitive ones (with the assumption that $1, \alpha, \beta$ are rationally independent).

\section{BOUNDED NUMBER OF LENGTHS}

This section is devoted to the proof of Statement (i) of Theorem 1.1. We provide a strategy for constructing examples of pairs $(\alpha, \beta)$ providing a low number of distances $\Delta\left(E_{N}(\alpha, \beta)\right)$, for all $N$. We will rely on Lemma 2.1, and use the existence of positive integers $b$ such that $q_{i}=b q_{j}^{\prime}+1$, as well as the existence of positive integers $b^{\prime}$ such that $q_{i}^{\prime}=b^{\prime} q_{j}+1$ for suitable $i, j$, with $\alpha$ and $\beta$ playing a symmetrical role. 


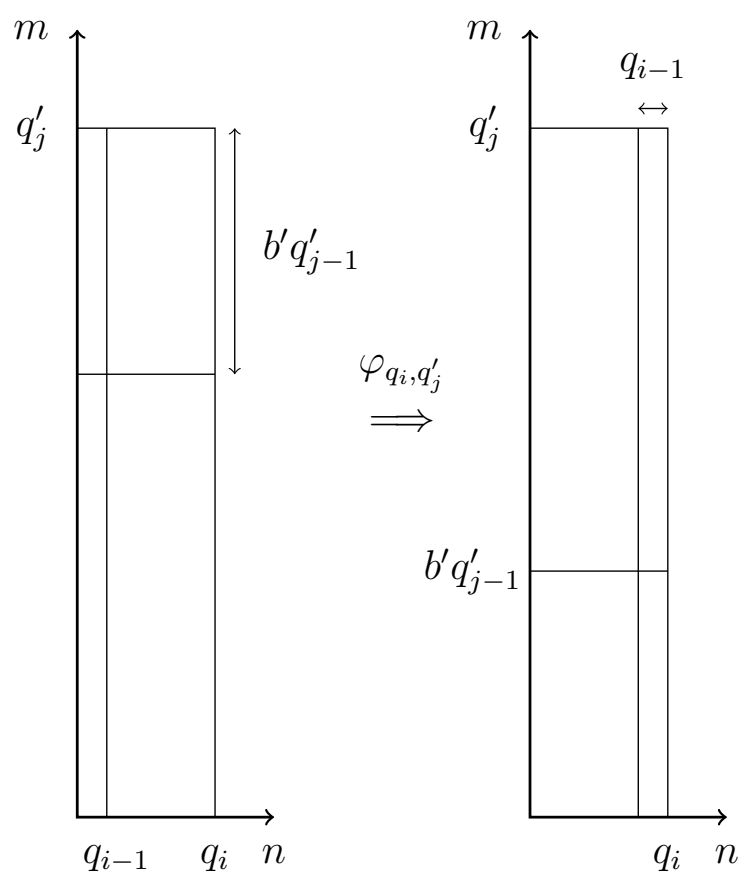

FIGURE 1. The action of $\varphi_{q_{i}, q_{j}^{\prime}}$ on $\mathcal{E}_{q_{i}, q_{j}^{\prime}}(\alpha, \beta)$ is an exchange of 4 sub-rectangles (here, $i$ and $j$ are assumed to be even).

Construction of the sequences of convergents $\left(q_{k}\right)_{k}$ and $\left(q_{k}^{\prime}\right)_{k}$. We provide a construction of sequences of convergents $\left(q_{k}\right)_{k},\left(q_{k}^{\prime}\right)_{k}$, and sequences $\left(b_{k}\right)_{k},\left(b_{k}^{\prime}\right)_{k}$ such that the following holds, for all $k \geq 1$ :

$$
q_{k}^{\prime}=b_{k}^{\prime} q_{k}+1, \quad q_{k+1}=b_{k+1} q_{k}^{\prime}+1 .
$$

Recall that $q_{-1}=q_{-1}^{\prime}=0$ and $q_{0}=q_{0}^{\prime}=1$. We then start with $q_{1}=3$, $q_{1}^{\prime}=\left(q_{1}\right)^{3}+1=28$ with $b_{1}^{\prime}=9$. Also $a_{1}=q_{1}=3, a_{1}^{\prime}=q_{1}^{\prime}=28$. Let

$$
a_{2}=\left(\left(q_{1}\right)^{6}+q_{0}-1\right) b_{1}^{\prime}+\left(q_{1}\right)^{5}=3^{8}+3^{5}, \quad q_{2}=3^{9}+3^{6}+1=3^{6} q_{1}^{\prime}+1 .
$$

We set $b_{2}=3^{6}=\left(q_{1}\right)^{6}+q_{0}-1$.

Assume now that for some index $k$, one has $q_{k}^{\prime}=b_{k}^{\prime} q_{k}+1$. Choose $a_{k+1}=\left(\left(q_{k}\right)^{6}+q_{k-1}-1\right) b_{k}^{\prime}+\left(q_{k}\right)^{5}$. Then, we get

$$
\begin{aligned}
q_{k+1} & =a_{k+1} q_{k}+q_{k-1}=\left(\left(q_{k}\right)^{6}+q_{k-1}-1\right) b_{k}^{\prime} q_{k}+\left(q_{k}\right)^{6}+q_{k-1} \\
& =\left(\left(q_{k}\right)^{6}+q_{k-1}-1\right)\left(b_{k}^{\prime} q_{k}+1\right)+1=\left(\left(q_{k}\right)^{6}+q_{k-1}-1\right) q_{k}^{\prime}+1 .
\end{aligned}
$$

Let $b_{k+1}=\left(q_{k}\right)^{6}+q_{k-1}-1$. Then $q_{k+1}=b_{k+1} q_{k}^{\prime}+1$. Next, we set $a_{k+1}^{\prime}=$ $\left(\left(q_{k}^{\prime}\right)^{6}+q_{k-1}^{\prime}-1\right) b_{k+1}+\left(q_{k}^{\prime}\right)^{5}$. Then, we have similarly

$$
\begin{aligned}
q_{k+1}^{\prime} & =a_{k+1}^{\prime} q_{k}^{\prime}+q_{k-1}^{\prime}=\left(\left(q_{k}^{\prime}\right)^{6}+q_{k-1}^{\prime}-1\right) b_{k+1} q_{k}^{\prime}+\left(q_{k}^{\prime}\right)^{6}+q_{k-1}^{\prime} \\
& =\left(\left(q_{k}^{\prime}\right)^{6}+q_{k-1}^{\prime}-1\right)\left(b_{k+1} q_{k}^{\prime}+1\right)+1=\left(\left(q_{k}^{\prime}\right)^{6}+q_{k-1}^{\prime}-1\right) q_{k+1}+1
\end{aligned}
$$

and $b_{k+1}^{\prime}=\left(q_{k}^{\prime}\right)^{6}+q_{k-1}^{\prime}-1$. 
In summary, we inductively construct sequences $\left(q_{k}\right)_{k},\left(q_{k}^{\prime}\right)_{k}$ satisfying for any $k \geq 1$

$$
\begin{gathered}
a_{k+1}=\left(\left(q_{k}\right)^{6}+q_{k-1}-1\right) b_{k}^{\prime}+\left(q_{k}\right)^{5}, \\
a_{k+1}^{\prime}=\left(\left(q_{k}^{\prime}\right)^{6}+q_{k-1}^{\prime}-1\right) b_{k+1}+\left(q_{k}^{\prime}\right)^{5}, \\
b_{k+1}=\left(q_{k}\right)^{6}+q_{k-1}-1, \quad b_{k+1}^{\prime}=\left(q_{k}^{\prime}\right)^{6}+q_{k-1}^{\prime}-1 .
\end{gathered}
$$

Then, for any $k \geq 1$, (3.1) holds. Note that

$$
q_{k+1}=b_{k+1} q_{k}^{\prime}+1=b_{k+1}\left(b_{k}^{\prime} q_{k}+1\right)+1=b_{k}^{\prime}\left(b_{k+1} q_{k}\right)+\left(b_{k+1}+1\right) .
$$

Since

$$
\begin{aligned}
a_{k+1} & =\frac{q_{k+1}-q_{k-1}}{q_{k}}=\frac{b_{k+1} q_{k}^{\prime}+1-q_{k-1}}{q_{k}} \\
& =\frac{\left(\left(q_{k}\right)^{6}+q_{k-1}-1\right) q_{k}^{\prime}+1-q_{k-1}}{q_{k}} \geq\left(q_{k}\right)^{5} q_{k}^{\prime},
\end{aligned}
$$

we have

$$
q_{k-1}\left\|q_{k} \alpha\right\|<\frac{1}{a_{k+1} a_{k}}<\frac{1}{2 q_{k}^{\prime}}<\left\|q_{k-1}^{\prime} \beta\right\|<\left\|q_{k-1}^{\prime} \beta\right\|+q_{k-1}^{\prime}\left\|q_{k}^{\prime} \beta\right\| .
$$

Therefore, it follows that

$$
\begin{aligned}
\left\|q_{k-1} \alpha\right\|-b_{k}^{\prime}\left\|q_{k-1}^{\prime} \beta\right\| & >\frac{1-q_{k-1}\left\|q_{k} \alpha\right\|}{q_{k}}-\frac{b_{k}^{\prime}}{q_{k}^{\prime}} \\
& =\frac{q_{k}^{\prime}-b_{k}^{\prime} q_{k}}{q_{k} q_{k}^{\prime}}-\frac{q_{k-1}}{q_{k}}\left\|q_{k} \alpha\right\|=\frac{1}{q_{k} q_{k}^{\prime}}-\frac{q_{k-1}}{q_{k}}\left\|q_{k} \alpha\right\| \\
& =\frac{q_{k+1}\left\|q_{k} \alpha\right\|+q_{k}\left\|q_{k+1} \alpha\right\|}{q_{k} q_{k}^{\prime}}-\frac{q_{k-1}}{q_{k}}\left\|q_{k} \alpha\right\| \\
& =\left(\frac{b_{k+1}}{q_{k}}+\frac{1}{q_{k} q_{k}^{\prime}}-\frac{q_{k-1}}{q_{k}}\right)\left\|q_{k} \alpha\right\|+\frac{\left\|q_{k+1} \alpha\right\|}{q_{k}^{\prime}} \\
& =\left(\left(q_{k}\right)^{5}-\frac{1}{q_{k}}+\frac{1}{q_{k} q_{k}^{\prime}}\right)\left\|q_{k} \alpha\right\|+\frac{\left\|q_{k+1} \alpha\right\|}{q_{k}^{\prime}}>0 .
\end{aligned}
$$

We also claim that

$$
q_{k}<b_{k}^{\prime}<\left(q_{k}\right)^{3}, \quad q_{k}^{\prime}<b_{k+1}<\left(q_{k}^{\prime}\right)^{3} .
$$

Indeed, if $q_{k}<b_{k}^{\prime}<\left(q_{k}\right)^{3}$, then, using (3.3), we have

$$
q_{k}^{\prime}=b_{k}^{\prime} q_{k}+1<q_{k}^{4}+1<b_{k+1}<\left(q_{k}^{2}+1\right)^{3}<\left(b_{k}^{\prime} q_{k}+1\right)^{3}=\left(q_{k}^{\prime}\right)^{3} .
$$

The choice of $q_{1}, q_{1}^{\prime}, b_{1}$ with $q_{1}<b_{1}^{\prime}<q_{1}^{3}$ concludes the proof of the claim. 
Rational independence of $1, \alpha, \beta$. Suppose that $1, \alpha, \beta$ are rationally dependent. Then, there exist integers $n_{0}, n_{1}, n_{2}$ satisfying $n_{0}+n_{1} \alpha+n_{2} \beta=0$. Since $\alpha, \beta$ are both irrational numbers, one has $n_{1}, n_{2} \neq 0$. Then, for large $k$ such that

$$
\left|n_{1}\right|<\frac{q_{k+1}}{q_{k}^{\prime}} \text { and }\left|n_{2}\right|<\frac{b_{k+1}^{\prime}}{2}<b_{k+1}^{\prime} q_{k+1}\left\|q_{k} \alpha\right\|<q_{k+1}^{\prime}\left\|q_{k} \alpha\right\|
$$

we have

$$
\left\|n_{1} q_{k}^{\prime} \alpha\right\|=\left\|n_{2} q_{k}^{\prime} \beta\right\| \leq\left|n_{2}\right|\left\|q_{k}^{\prime} \beta\right\|<\frac{\left|n_{2}\right|}{q_{k+1}^{\prime}}<\left\|q_{k} \alpha\right\| .
$$

This is a contradiction to the fact that $\|n \alpha\|>\left\|q_{k} \alpha\right\|$ for any $1 \leq n<q_{k+1}$ (see for instance [Lan95, Chapter 1, Theorem 6]).

Let us check now that $(\alpha, \beta)$ is not badly approximable. Recall that an irrational vector $(\alpha, \beta)$ is said to be badly approximable if there exists $C>0$ such that

$$
\|n \alpha+m \beta\|>\frac{C}{|(n, m)|^{2}}
$$

for any non-zero pair of integers $(n, m)$. For the example constructed in this section, if $(n, m)=\left(q_{k}, 0\right)$, then, by (3.4), one gets

$$
\left\|q_{k} \alpha+0 \beta\right\|=\left\|q_{k} \alpha\right\|<\frac{1}{q_{k+1}}<\frac{1}{b_{k}^{\prime} b_{k+1} q_{k}}<\frac{1}{\left(q_{k}\right)^{7}}=\frac{1}{\left(q_{k}\right)^{5}} \frac{1}{\left|\left(q_{k}, 0\right)\right|^{2}} .
$$

Therefore, $(\alpha, \beta)$ is not badly approximable.

Organization of the proof. We first assume $q_{k}<N \leq q_{k}^{\prime}$ and $k$ is even, and provide all the details for this case. The case $k$ odd and then, the case $q_{k}<N \leq q_{k}^{\prime}$, will be briefly discussed at the end of the proof.

We thus assume $q_{k}<N \leq q_{k}^{\prime}$ and $k$ is even (see Figure 2). Note that $q_{k}^{\prime}=b_{k}^{\prime} q_{k}+1>q_{k}$. The proof will be divided into three steps.

- We first describe the lengths in $E_{q_{k}, q_{k}^{\prime}}(\alpha, \beta)$. There are 4 lengths according to Lemma 2.1 .

- Then, we will deduce the description of the lengths in $E_{q_{k}, N}(\alpha, \beta)$ from the description of the lengths in $E_{q_{k}, q_{k}^{\prime}}(\alpha, \beta)$. We reduce the set of points $(n, m)$ under consideration in this step. Dynamically, this will correspond to induce the map $\phi_{q_{k}, q_{k}^{\prime}}$ (or similarly the map $\left.\varphi_{q_{k}, q_{k}^{\prime}}\right)$. We will go from 4 lengths to 6 lengths.

- Lastly, the description of the lengths in $E_{N}(\alpha, \beta)$ will be deduced from the description of the lengths in $E_{q_{k}, N}(\alpha, \beta)$ by performing an 'exduction' step with points $(n, m)$ being inserted, creating a seventh length. 


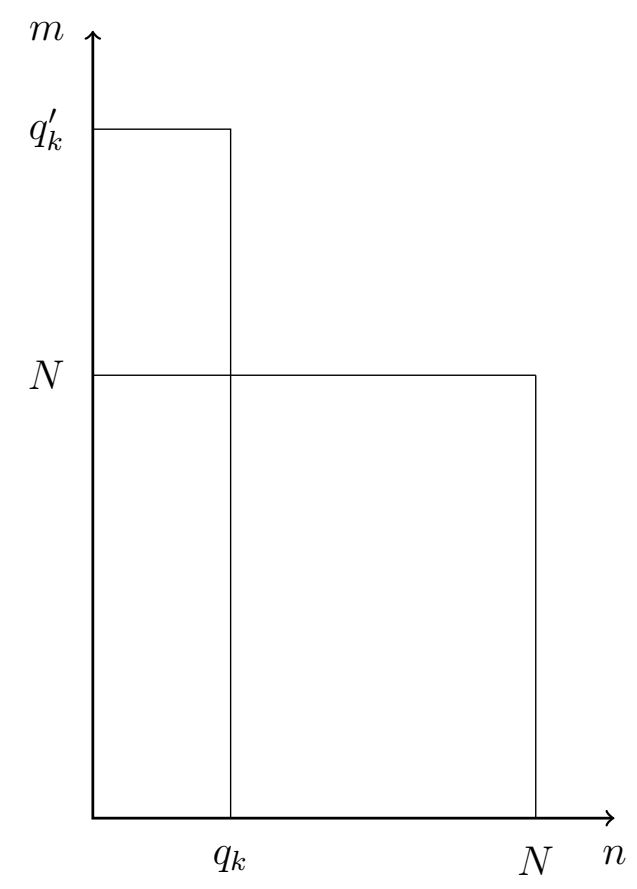

Figure 2. The sets $\mathcal{E}_{q_{k}, q_{k}^{\prime}}, \mathcal{E}_{q_{k}, N}$ and $\mathcal{E}_{N}$.

Distribution of the points of $E_{q_{k}, q_{k}^{\prime}}(\alpha, \beta)$. We apply Lemma 2.1 for $E_{q_{k}, q_{k}^{\prime}}(\alpha, \beta)$ by using the fact that $q_{k}^{\prime}=b_{k}^{\prime} q_{k}+1$. With the notation of the lemma, $i=j=k, b=b_{k}^{\prime}$. We are in the case $i, j$ even, since $k$ is even. Observe that Assumption (2.3) holds, namely $\left\|q_{k}^{\prime} \beta\right\|<\left\|q_{k-1} \alpha\right\|-b_{k}^{\prime}\left\|q_{k-1}^{\prime} \beta\right\|$. This comes from (3.5), applied twice to get the two left inequalities below:

$$
\left\|q_{k-1} \alpha\right\|-b_{k}^{\prime}\left\|q_{k-1}^{\prime} \beta\right\|>\left\|q_{k} \alpha\right\|>b_{k+1}^{\prime}\left\|q_{k}^{\prime} \beta\right\| \geq\left\|q_{k}^{\prime} \beta\right\| .
$$

By Lemma 2.1, the neighbor map $\Phi_{q_{k}, q_{k}^{\prime}}$ on $E_{q_{k}, q_{k}^{\prime}}(\alpha, \beta)$ satisfies

$$
\Phi_{q_{k}, q_{k}^{\prime}}: n \alpha+m \beta \mapsto\left|n-q_{k-1}\right|_{q_{k}} \alpha+\left|m+b_{k}^{\prime} q_{k-1}^{\prime}\right|_{q_{k}^{\prime}} \beta
$$

and

$$
\begin{aligned}
& \Delta_{q_{k}, q_{k}^{\prime}}(n, m)= \\
& \begin{cases}\left\|q_{k-1} \alpha\right\|-b_{k}^{\prime}\left\|q_{k-1}^{\prime} \beta\right\|, & q_{k-1} \leq n, m<q_{k}^{\prime}-b_{k}^{\prime} q_{k-1}^{\prime}, \\
\left\|q_{k-1} \alpha\right\|-b_{k}^{\prime}\left\|q_{k-1}^{\prime} \beta\right\|-\left\|q_{k}^{\prime} \beta\right\|, & q_{k-1} \leq n, q_{k}^{\prime}-b_{k}^{\prime} q_{k-1}^{\prime} \leq m, \\
\left\|q_{k-1} \alpha\right\|-b_{k}^{\prime}\left\|q_{k-1}^{\prime} \beta\right\|+\left\|q_{k} \alpha\right\|, & n<q_{k-1}, m<q_{k}^{\prime}-b_{k}^{\prime} q_{k-1}^{\prime}, \\
\left\|q_{k-1} \alpha\right\|-b_{k}^{\prime}\left\|q_{k-1}^{\prime} \beta\right\|+\left\|q_{k} \alpha\right\|-\left\|q_{k}^{\prime} \beta\right\|, & n<q_{k-1}, \quad q_{k}^{\prime}-b_{k}^{\prime} q_{k-1}^{\prime} \leq m .\end{cases}
\end{aligned}
$$

Let $\varphi_{q_{k}, q_{k}^{\prime}}$ be the map defined on $\mathcal{E}_{q_{k}, q_{k}^{\prime}}$ as $\varphi_{q_{k}, q_{k}^{\prime}}(n, m)=\left(\left|n-q_{k-1}\right|_{q_{k}}, \mid m+\right.$ $\left.\left.b_{k}^{\prime} q_{k-1}^{\prime}\right|_{q_{k}^{\prime}}\right)$. Its action is shown in Figure 3 (on the left) as an exchange of 4 sub-rectangles. Recall that $\varphi_{q_{k}, q_{k}^{\prime}}$ and $\Phi_{q_{k}, q_{k}^{\prime}}$ are related by (2.1). 
Distribution of the points of $E_{q_{k}, N}(\alpha, \beta)$ for $q_{k}<N \leq q_{k}^{\prime}$. We obtain $\Phi_{q_{k}, N}$ on $E_{q_{k}, N}(\alpha, \beta)$ by iterating the map $\Phi_{q_{k}, q_{k}^{\prime}}$. Indeed, since $E_{q_{k}, N}(\alpha, \beta)$ is a subset of $E_{q_{k}, q_{k}^{\prime}}(\alpha, \beta)$, the neighbor map $\Phi_{q_{k}, N}$ on $E_{q_{k}, N}(\alpha, \beta)$ is the induced map of $\Phi_{q_{k}, q_{k}^{\prime}}$ on $E_{q_{k}, N}(\alpha, \beta)$, i.e.,

$$
\Phi_{q_{k}, N}(x)=\left(\Phi_{q_{k}, q_{k}^{\prime}}\right)^{\tau(x)}(x),
$$

where $\tau(x)=\min \left\{\ell \geq 1:\left(\Phi_{q_{k}, q_{k}^{\prime}}\right)^{\ell}(x) \in E_{q_{k}, N}(\alpha, \beta)\right\}$ is the first return time to $E_{q_{k}, N}(\alpha, \beta)$ of the map $\Phi_{q_{k}, q_{k}^{\prime}}$. This induction step will create two more sub-rectangles, that is, $\varphi_{q_{k}, q_{k}^{\prime}}$ acts as an exchange of 4 sub-rectangles, while $\varphi_{q_{k}, N}$ acts as an exchange of 6 sub-rectangles (see Figure 3).

The following lemma expresses the fact that the return time $\tau$ takes 3 values. Note that the statement below does not depend on the parity of $k$.

Lemma 3.1. Let $\tau$ be the first return time to $E_{q_{k}, N}(\alpha, \beta)$ of the map $\Phi_{q_{k}, q_{k}^{\prime}}$. There exist $\tau_{1}, \tau_{2}, \tau_{3}, N_{1}, N_{2}, N_{3}$ such that, for each $n \alpha+m \beta \in E_{q_{k}, N}(\alpha, \beta)$, we have

$$
\tau(n \alpha+m \beta)= \begin{cases}\tau_{1}, & \text { if } 0 \leq m<N_{1}, \\ \tau_{1}+\tau_{2}, & \text { if } N_{1} \leq m<N_{2}, \\ \tau_{2}, & \text { if } N_{2} \leq m<N .\end{cases}
$$

Moreover, there exist $d_{1}, d_{2}$ nonnegative integers such that

$$
\begin{aligned}
{\left[0, N_{1}\right)+\tau_{1} b_{k}^{\prime} q_{k-1}^{\prime} } & =\left[N-N_{1}, N\right)+d_{1} q_{k}^{\prime}, \\
{\left[N_{1}, N_{2}\right)+\left(\tau_{1}+\tau_{2}\right) b_{k}^{\prime} q_{k-1}^{\prime} } & =\left[N-N_{2}, N-N_{1}\right)+\left(d_{1}+d_{2}\right) q_{k}^{\prime}, \\
{\left[N_{2}, N\right)+\tau_{2} b_{k}^{\prime} q_{k-1}^{\prime} } & =\left[0, N-N_{2}\right)+d_{2} q_{k}^{\prime} .
\end{aligned}
$$

Proof. We prove the lemma for $k$ even, but the same argument works for $k$ odd. Recall that, for even $k, \Phi_{q_{k}, q_{k}^{\prime}}(n \alpha+m \beta)=\left|n-q_{k-1}\right|_{q_{k}} \alpha+\mid m+$ $\left.b_{k}^{\prime} q_{k-1}^{\prime}\right|_{q_{k}^{\prime}} \beta$. Thus

$$
\left(\Phi_{q_{k}, q_{k}^{\prime}}\right)^{\ell}(n \alpha+m \beta) \in E_{q_{k}, N}(\alpha, \beta) \text { if and only if } 0 \leq\left|m+\ell b_{k}^{\prime} q_{k-1}^{\prime}\right|_{q_{k}^{\prime}}<N .
$$

Let

$$
\bar{\tau}(m)=\min \left\{\ell \geq 1: 0 \leq\left|m+\ell b_{k}^{\prime} q_{k-1}^{\prime}\right|_{q_{k}^{\prime}}<N\right\} .
$$

The discrete version of the three-gap problem (see e.g. [Sla67]) applied to the translation by $b_{k}^{\prime} q_{k-1}^{\prime}$ modulo $N$ provides the existence of $\tau_{1}, \tau_{2}, \tau_{3}$, $N_{1}, N_{2}, N_{3}$ such that

$$
\bar{\tau}(m)= \begin{cases}\tau_{1}, & \text { if } 0 \leq m<N_{1} \\ \tau_{1}+\tau_{2}, & \text { if } N_{1} \leq m<N_{2}, \\ \tau_{2}, & \text { if } N_{2} \leq m<N\end{cases}
$$


as well as the existence of nonnegative integers $d_{1}, d_{2}$ satisfying

$$
\begin{aligned}
{\left[0, N_{1}\right)+\tau_{1} b_{k}^{\prime} q_{k-1}^{\prime} } & =\left[N-N_{1}, N\right)+d_{1} q_{k}^{\prime}, \\
{\left[N_{2}, N\right)+\tau_{2} b_{k}^{\prime} q_{k-1}^{\prime} } & =\left[0, N-N_{2}\right)+d_{2} q_{k}^{\prime} .
\end{aligned}
$$

Clearly, we have

$$
\left[N_{1}, N_{2}\right)+\left(\tau_{1}+\tau_{2}\right) b_{k}^{\prime} q_{k-1}^{\prime}=\left[N-N_{2}, N-N_{1}\right)+\left(d_{1}+d_{2}\right) q_{k}^{\prime} .
$$

Lemma 3.1 is thus a direct consequence of the discrete three-gap problem.

Therefore, for $k$ even, we deduce from Lemma 3.1 that

$$
\begin{aligned}
& \Phi_{q_{k}, N}(n \alpha+m \beta)=\left(\Phi_{q_{k}, q_{k}^{\prime}}\right)^{\tau(n \alpha+m \beta)}(n \alpha+m \beta) \\
& =\left\{\begin{array}{cll}
\left|n-\tau_{1} q_{k-1}\right|_{q_{k}} \alpha+\left(m+\tau_{1} b_{k}^{\prime} q_{k-1}^{\prime}-d_{1} q_{k}^{\prime}\right) \beta, & 0 \leq m<N_{1}, \\
\left|n-\left(\tau_{1}+\tau_{2}\right) q_{k-1}\right|_{q_{k}} \alpha & \\
\quad+\left(m+\left(\tau_{1}+\tau_{2}\right) b_{k}^{\prime} q_{k-1}^{\prime}-\left(d_{1}+d_{2}\right) q_{k}^{\prime}\right) \beta, & N_{1} \leq m<N_{2}, \\
\left|n-\tau_{2} q_{k-1}\right|_{q_{k}} \alpha+\left(m+\tau_{2} b_{k}^{\prime} q_{k-1}^{\prime}-d_{2} q_{k}^{\prime}\right) \beta, & N_{2} \leq m<N .
\end{array}\right.
\end{aligned}
$$

Let $h_{1}, h_{2}, h_{3}$ be nonnegative integers satisfying

$$
\tau_{1} q_{k-1}=h_{1} q_{k}+r_{1}, \quad \tau_{2} q_{k-1}=h_{2} q_{k}+r_{2}, \quad\left(\tau_{1}+\tau_{2}\right) q_{k-1}=h_{3} q_{k}+r_{3}
$$

with $0 \leq r_{1}, r_{2}, r_{3}<q_{k}$. Each of the three cases splits into two cases according to the fact that $n$ is smaller or not than $r_{i}$, for $i=1,2,3$. Then, we have

$$
\Delta_{q_{k}, N}(n, m)= \begin{cases}\Delta_{1}+\left\|q_{k} \alpha\right\|, & \text { if } 0 \leq n<r_{1}, 0 \leq m<N_{1}, \\ \Delta_{1}, & \text { if } r_{1} \leq n<q_{k}, 0 \leq m<N_{1}, \\ \Delta_{3}+\left\|q_{k} \alpha\right\|, & \text { if } 0 \leq n<r_{3}, N_{1} \leq m<N_{2}, \\ \Delta_{3}, & \text { if } r_{3} \leq n<q_{k}, N_{1} \leq m<N_{2}, \\ \Delta_{2}+\left\|q_{k} \alpha\right\|, & \text { if } 0 \leq n<r_{2}, N_{2} \leq m<N, \\ \Delta_{2}, & \text { if } r_{2} \leq n<q_{k}, N_{2} \leq m<N,\end{cases}
$$

where

$$
\begin{aligned}
& \Delta_{1}=\tau_{1}\left(\left\|q_{k-1} \alpha\right\|-b_{k}^{\prime}\left\|q_{k-1}^{\prime} \beta\right\|\right)-d_{1}\left\|q_{k}^{\prime} \beta\right\|+h_{1}\left\|q_{k} \alpha\right\|, \\
& \Delta_{2}=\tau_{2}\left(\left\|q_{k-1} \alpha\right\|-b_{k}^{\prime}\left\|q_{k-1}^{\prime} \beta\right\|\right)-d_{2}\left\|q_{k}^{\prime} \beta\right\|+h_{2}\left\|q_{k} \alpha\right\|, \\
& \Delta_{3}=\left(\tau_{1}+\tau_{2}\right)\left(\left\|q_{k-1} \alpha\right\|-b_{k}^{\prime}\left\|q_{k-1}^{\prime} \beta\right\|\right)-\left(d_{1}+d_{2}\right)\left\|q_{k}^{\prime} \beta\right\|+h_{3}\left\|q_{k} \alpha\right\| .
\end{aligned}
$$



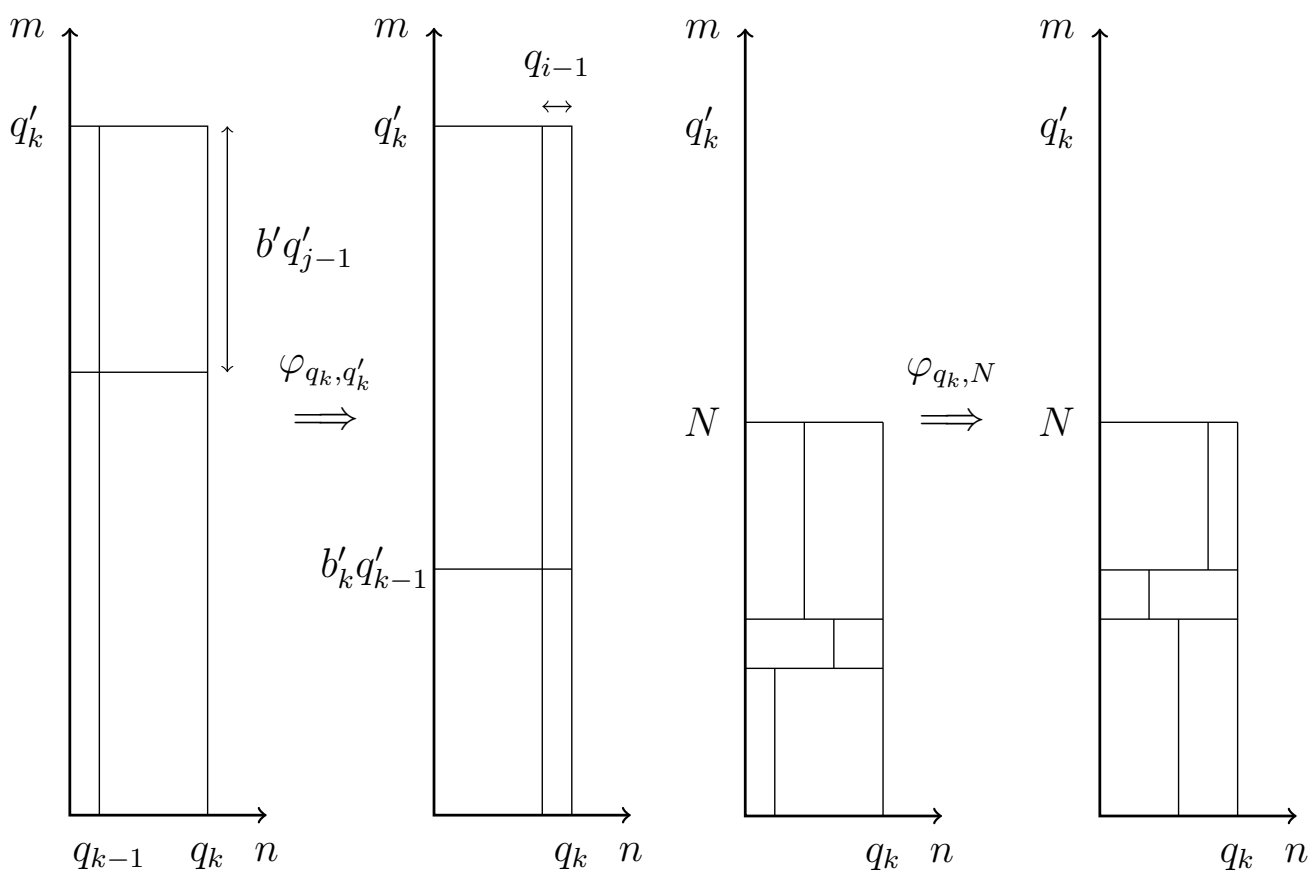

Figure 3. The action of $\varphi_{q_{k}, q_{k}^{\prime}}$ on $\mathcal{E}_{q_{k}, q_{k}^{\prime}}(\alpha, \beta)$ is an exchange of 4 sub-rectangles (left), and the action of $\varphi_{q_{k}, N}$ on $\mathcal{E}_{q_{k}, N}(\alpha, \beta)$ is an exchange of 6 sub-rectangles (right).

Indeed, in the case where, for example, $0 \leq m<N_{1}$, we have, by (3.7),

$$
\begin{aligned}
& \Phi_{q_{k}, N}(n \alpha+m \beta)-(n \alpha+m \beta) \\
& \quad=\left|n-\tau_{1} q_{k-1}\right|_{q_{k}} \alpha+\left(m+\tau_{1} b_{k}^{\prime} q_{k-1}^{\prime}-d_{1} q_{k}^{\prime}\right) \beta-(n \alpha+m \beta) \\
& =\left(\left|n-r_{1}\right|_{q_{k}}-n\right) \alpha+\left(\tau_{1} b_{k}^{\prime} q_{k-1}^{\prime}-d_{1} q_{k}^{\prime}\right) \beta \\
& = \begin{cases}\left(-r_{1}+q_{k}\right) \alpha+\left(\tau_{1} b_{k}^{\prime} q_{k-1}^{\prime}-d_{1} q_{k}^{\prime}\right) \beta, & \text { if } n<r_{1}, \\
-r_{1} \alpha+\left(\tau_{1} b_{k}^{\prime} q_{k-1}^{\prime}-d_{1} q_{k}^{\prime}\right) \beta, & \text { if } n \geq r_{1},\end{cases}
\end{aligned}
$$

and

$$
\begin{array}{r}
-r_{1} \alpha+\left(\tau_{1} b_{k}^{\prime} q_{k-1}^{\prime}-d_{1} q_{k}^{\prime}\right) \beta=\left(h_{1} q_{k}-\tau_{1} q_{k-1}\right) \alpha+\left(\tau_{1} b_{k}^{\prime} q_{k-1}^{\prime}-d_{1} q_{k}^{\prime}\right) \beta \\
=\tau_{1}\left(\left\|q_{k-1} \alpha\right\|-b_{k}^{\prime}\left\|q_{k-1}^{\prime} \beta\right\|\right)-d_{1}\left\|q_{k}^{\prime} \beta\right\|+h_{1}\left\|q_{k} \alpha\right\|=\Delta_{1} .
\end{array}
$$

The action of $\varphi_{q_{k}, N}(n, m)=\left(\varphi_{q_{k}, q_{k}^{\prime}}\right)^{\tau(m)}(n, m)$ on $\mathcal{E}_{q_{k}, N}$ is illustrated in Figure 3 .

From $E_{q_{k}, N}(\alpha, \beta)$ to $E_{N}(\alpha, \beta)$. Let $N=a q_{k}+R$, with $a \geq 1$ and $1 \leq R \leq$ $q_{k}$ (recall that $q_{k}<N \leq q_{k}^{\prime}$ ). Since $E_{q_{k}, N}$ is a subset of $E_{q_{k}, q_{k}^{\prime}}$, we have

$$
\min \Delta\left(E_{q_{k}, N}\right) \geq \min \Delta\left(E_{q_{k}, q_{k}^{\prime}}\right)=\left\|q_{k-1} \alpha\right\|-b_{k}^{\prime}\left\|q_{k-1}^{\prime} \beta\right\|-\left\|q_{k}^{\prime} \beta\right\| .
$$




$$
\underset{n \alpha+m \beta \quad}{\bullet\left(n+2 q_{k}\right) \alpha+m \beta \cdots\left(n+a q_{k}\right) \alpha+m \beta \quad} \quad \cdots \quad-
$$

Figure 4. Illustration of the proof of Claim (3.9), when $0 \leq$ $n<R$.

Using (3.5) and (3.6), it follows that

$$
\begin{aligned}
\min \Delta\left(E_{q_{k}, N}\right) & \geq\left\|q_{k-1} \alpha\right\|-b_{k}^{\prime}\left\|q_{k-1}^{\prime} \beta\right\|-\left\|q_{k}^{\prime} \beta\right\| \\
& >\left(\left(q_{k}\right)^{5}-\frac{1}{q_{k}}\right)\left\|q_{k} \alpha\right\|-\frac{\left\|q_{k} \alpha\right\|}{b_{k+1}^{\prime}}>\left(\left(q_{k}\right)^{3}+1\right)\left\|q_{k} \alpha\right\| \\
& >\frac{b_{k}^{\prime} q_{k}+1}{q_{k}}\left\|q_{k} \alpha\right\|=\frac{q_{k}^{\prime}}{q_{k}}\left\|q_{k} \alpha\right\| \geq \frac{N}{q_{k}}\left\|q_{k} \alpha\right\|>a\left\|q_{k} \alpha\right\| .
\end{aligned}
$$

We claim that

$$
\Phi_{N}(n \alpha+m \beta)= \begin{cases}\left(n+q_{k}\right) \alpha+m \beta, & \text { if } 0 \leq n<N-q_{k}, \\ \Phi_{q_{k}, N}\left(|n|_{q_{k}} \alpha+m \beta\right), & \text { if } N-q_{k} \leq n<N .\end{cases}
$$

Proof of Claim (3.9). If $0 \leq n<R=N-a q_{k}$, then the points $\left(n+q_{k}\right) \alpha+$ $m \beta,\left(n+2 q_{k}\right) \alpha+m \beta, \ldots,\left(n+a q_{k}\right) \alpha+m \beta$ are located between $n \alpha+m \beta$ and $\Phi_{q_{k}, N}(n \alpha+m \beta)$, as shown in Figure 4. Therefore, we have $\Delta_{N}\left(n+c q_{k}, m\right)=$ $\left\|q_{k} \alpha\right\|$, for $0 \leq c \leq a-1$, and $\Delta_{N}\left(n+a q_{k}, m\right)=\Delta_{q_{k}, N}(n, m)-a\left\|q_{k} \alpha\right\|$, which is positive by (3.8).

If $R \leq n<q_{k}$, then the points $\left(n+q_{k}\right) \alpha+m \beta,\left(n+2 q_{k}\right) \alpha+m \beta, \ldots$, $\left(n+(a-1) q_{k}\right) \alpha+m \beta$ are located between $n \alpha+m \beta$ and $\Phi_{q_{k}, N}(n \alpha+m \beta)$. In this case, the gaps between two adjacent points of $E_{N}(\alpha, \beta)$ are given by $\Delta_{N}\left(n+c q_{k}, m\right)=\left\|q_{k} \alpha\right\|$, for $0 \leq c \leq a-2$, and $\Delta_{N}\left(n+(a-1) q_{k}, m\right)=$ $\Delta_{q_{k}, N}(n, m)-(a-1)\left\|q_{k} \alpha\right\|$, which is positive by (3.8).

Therefore, using (3.7), we deduce that

$$
\begin{aligned}
& \Phi_{N}(n \alpha+m \beta) \\
& = \begin{cases}\left(n+q_{k}\right) \alpha+m \beta, & 0 \leq n<N-q_{k}, \\
\left|n-\tau_{1} q_{k-1}\right|_{q_{k}} \alpha+\left(m+\tau_{1} b_{k}^{\prime} q_{k-1}^{\prime}-d_{1} q_{k}^{\prime}\right) \beta, & n \geq N-q_{k}, 0 \leq m<N_{1}, \\
\left|n-\left(\tau_{1}+\tau_{2}\right) q_{k-1}\right|_{q_{k}} \alpha & \\
\quad+\left(m+\left(\tau_{1}+\tau_{2}\right) b_{k}^{\prime} q_{k-1}^{\prime}-\left(d_{1}+d_{2}\right) q_{k}^{\prime}\right) \beta, & n \geq N-q_{k}, N_{1} \leq m<N_{2}, \\
\left|n-\tau_{2} q_{k-1}\right|_{q_{k}} \alpha+\left(m+\tau_{2} b_{k}^{\prime} q_{k-1}^{\prime}-d_{2} q_{k}^{\prime}\right) \beta, & n \geq N-q_{k}, N_{2} \leq m<N .\end{cases}
\end{aligned}
$$

Let $\bar{h}_{1}, \bar{h}_{2}, \bar{h}_{3}$ be nonnegative integers satisfying

$$
N-\tau_{1} q_{k-1}=\bar{h}_{1} q_{k}+\bar{r}_{1}, N-\tau_{2} q_{k-1}=\bar{h}_{2} q_{k}+\bar{r}_{2}, N-\left(\tau_{1}+\tau_{2}\right) q_{k-1}=\bar{h}_{3} q_{k}+\bar{r}_{3}
$$



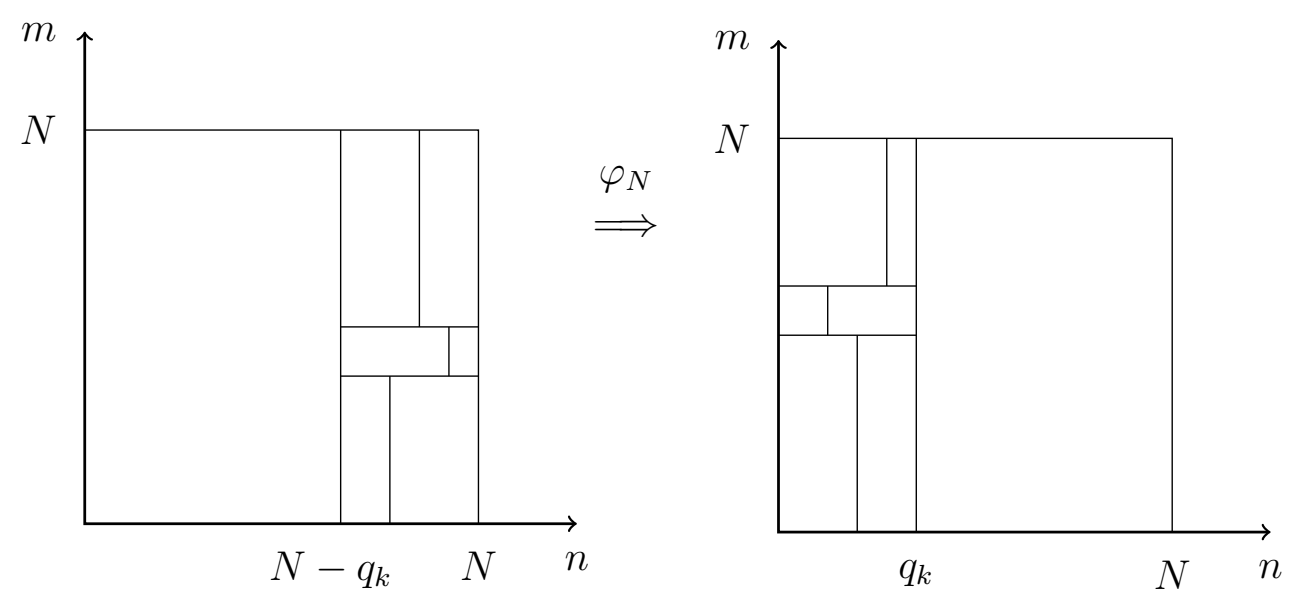

FiguRE 5. The action of $\varphi_{N}$ on $\mathcal{E}_{N}$ is an exchange of 7 sub-rectangles.

with $0 \leq \bar{r}_{1}, \bar{r}_{2}, \bar{r}_{3}<q_{k}$. Then, we have

$$
\Delta_{N}(n, m)= \begin{cases}\left\|q_{k} \alpha\right\|, & 0 \leq n<N-q_{k}, \\ \Delta_{1}+\left\|q_{k} \alpha\right\|, & N-q_{k} \leq n<N-\bar{r}_{1}, 0 \leq m<N_{1}, \\ \Delta_{1}, & N-\bar{r}_{1} \leq n<N, 0 \leq m<N_{1}, \\ \Delta_{3}+\left\|q_{k} \alpha\right\|, & N-q_{k} \leq n<N-r_{3}, \quad N_{1} \leq m<N_{2}, \\ \Delta_{3}, & N-\bar{r}_{3} \leq n<q_{k}, N_{1} \leq m<N_{2}, \\ \Delta_{2}+\left\|q_{k} \alpha\right\|, & N-q_{k} \leq n<N-\bar{r}_{2}, \quad N_{2} \leq m<N, \\ \Delta_{2}, & N-\bar{r}_{2} \leq n<N, N_{2} \leq m<N,\end{cases}
$$

where

$$
\begin{aligned}
& \Delta_{1}=\tau_{1}\left(\left\|q_{k-1} \alpha\right\|-b_{k}^{\prime}\left\|q_{k-1}^{\prime} \beta\right\|\right)-d_{1}\left\|q_{k}^{\prime} \beta\right\|-\bar{h}_{1}\left\|q_{k} \alpha\right\|, \\
& \Delta_{2}=\tau_{2}\left(\left\|q_{k-1} \alpha\right\|-b_{k}^{\prime}\left\|q_{k-1}^{\prime} \beta\right\|\right)-d_{2}\left\|q_{k}^{\prime} \beta\right\|-\bar{h}_{2}\left\|q_{k} \alpha\right\|, \\
& \Delta_{3}=\left(\tau_{1}+\tau_{2}\right)\left(\left\|q_{k-1} \alpha\right\|-b_{k}^{\prime}\left\|q_{k-1}^{\prime} \beta\right\|\right)-\left(d_{1}+d_{2}\right)\left\|q_{k}^{\prime} \beta\right\|-\bar{h}_{3}\left\|q_{k} \alpha\right\| .
\end{aligned}
$$

The action of $\varphi_{N}(n, m)$ on $\mathcal{E}_{N}$ is illustrated in Figure 5.

End of the proof. The case $q_{k}<N \leq q_{k}^{\prime}$ and $k$ even has thus been handled. In the case $k$ odd, $\Delta_{q_{k}, q_{k}^{\prime}}(n, m)$ still takes four values, as discussed in Lemma 2.1, namely

$$
\begin{array}{ll}
\left\|q_{k-1} \alpha\right\|-b_{k}^{\prime}\left\|q_{k-1} \beta\right\|, & \left\|q_{k-1} \alpha\right\|-b_{k}^{\prime}\left\|q_{k-1} \beta\right\|-\left\|q_{k}^{\prime} \beta\right\|, \\
\left\|q_{k-1} \alpha\right\|-b_{k}^{\prime}\left\|q_{k-1} \beta\right\|+\left\|q_{k} \alpha\right\|, & \left\|q_{k-1} \alpha\right\|-b_{k}^{\prime}\left\|q_{k-1} \beta\right\|+\left\|q_{k} \alpha\right\|-\left\|q_{k}^{\prime} \beta\right\| .
\end{array}
$$

We also have at most 6 values for $\Delta\left(E_{q_{k}, N}(\alpha, \beta)\right)$ which are obtained by considering the induced map of $\Phi_{q_{k}, q_{k}^{\prime}}$. Observe that $\Delta_{q_{k}, q_{k}^{\prime}}(n, m)$ takes the same values as in the case $q_{k}<N \leq q_{k}^{\prime}$ and $k$ even. It follows from Claim (3.9) that there are 7 values for $\Delta\left(E_{N}(\alpha, \beta)\right)$. 
Lastly, the case $q_{k}^{\prime}<N \leq q_{k+1}$ is similarly deduced by induction from the case $E_{q_{k}^{\prime}, q_{k+1}}(\alpha, \beta)$. This ends the proof of Theorem 1.1 (i).

Remark 3.2. Observe that, in continuation of Remark 2.3, there are here also 4 primitive lengths.

\section{UNBOUNDED NUMBER OF LENGTHS}

This section is devoted to the proof of Statement (ii) of Theorem 1.1. The strategy works as follows: one wants to regularly get indices $k$ for which $q_{k}=q_{k}^{\prime}+1$. This will imply that $q_{k}$ and $q_{k}^{\prime}$ are coprime and that they have the same size. This will allow us in particular to consider mainly the first level $E_{q_{k}, q_{k}^{\prime}}(\alpha, \beta)$ (and in fact even here $\left.E_{q_{4 k+1}, q_{4 k+1}^{\prime}}(\alpha, \beta)\right)$ ). Now, we provide a construction of $\alpha$ and $\beta$ for which $q_{4 k-3}+1=q_{4 k-3}^{\prime}$ and $q_{4 k-2}-1=q_{4 k-2}^{\prime}$, for all $k$. Furthermore, we will have $a_{4 k+1}^{\prime}=1$.

Construction of the sequences of convergents $\left(q_{i}\right)_{i}$ and $\left(q_{j}^{\prime}\right)_{j}$. We consider $\alpha$ and $\beta$ irrationals in $(0,1)$ with respective sequences of partial quotients $\left(a_{i}\right)_{i}$ and $\left(a_{j}^{\prime}\right)_{j}$ satisfying

$$
a_{1}=2, \quad a_{2}=2 \quad \text { and } \quad a_{1}^{\prime}=3, \quad a_{2}^{\prime}=1 .
$$

Then we have

$$
q_{0}=1, q_{1}=2, q_{2}=5, \quad q_{0}^{\prime}=1, q_{1}^{\prime}=3, q_{2}^{\prime}=4 .
$$

We now inductively define $\left(a_{i}\right)_{i},\left(a_{j}^{\prime}\right)_{j}$ as follows. Suppose that

$$
q_{4 k-3}+1=q_{4 k-3}^{\prime}, \quad q_{4 k-2}-1=q_{4 k-2}^{\prime} \quad \text { for } k \geq 1 .
$$

Furthermore, let $R_{k}:=q_{4 k-3}+1=q_{4 k-3}^{\prime}$ and $Q_{k}:=q_{4 k-2}-1=q_{4 k-2}^{\prime}$.

Let

$$
\begin{gathered}
a_{4 k-1}:=1, \quad a_{4 k}:=3, \quad a_{4 k+1}:=2 Q_{k}+R_{k}-1, \quad a_{4 k+2}:=6 Q_{k}+4 R_{k}, \\
a_{4 k-1}^{\prime}:=2, \quad a_{4 k}^{\prime}:=4 Q_{k}+3 R_{k}-2, \quad a_{4 k+1}^{\prime}:=1, \quad a_{4 k+2}^{\prime}:=6 Q_{k}+4 R_{k}-1 .
\end{gathered}
$$

Then we get

$$
\begin{aligned}
q_{4 k-1} & =Q_{k}+R_{k}, & q_{4 k-1}^{\prime} & =2 Q_{k}+R_{k}, \\
q_{4 k} & =4 Q_{k}+3 R_{k}+1, & q_{4 k}^{\prime} & =8 Q_{k}^{2}+\left(10 R_{k}-3\right) Q_{k}+3 R_{k}^{2}-2 R_{k},
\end{aligned}
$$

and

$$
q_{4 k+1}=R_{k+1}-1, \quad q_{4 k+1}^{\prime}=R_{k+1}, \quad q_{4 k+2}=Q_{k+1}+1, \quad q_{4 k+2}^{\prime}=Q_{k+1},
$$

where we put inductively

$$
\begin{aligned}
R_{k+1} & =8 Q_{k}^{2}+\left(10 R_{k}-1\right) Q_{k}+3 R_{k}^{2}-R_{k} \\
Q_{k+1} & =48 Q_{k}^{3}+\left(96 R_{k}-6\right) Q_{k}^{2}+\left(\begin{array}{c}
\left.43 R_{k}^{2}-5 R_{k}-2\right) Q_{k}+12 R_{k}^{3}-4 R_{k}^{2}-R_{k} \\
18
\end{array}\right.
\end{aligned}
$$


Rational independence of $1, \alpha, \beta$. Suppose that $1, \alpha, \beta$ are rationally dependent. Then, there exist integers $n_{0}, n_{1}, n_{2}$ satisfying $n_{0}+n_{1} \alpha+n_{2} \beta=0$. Since $\alpha, \beta$ are both irrational numbers, $n_{1}, n_{2} \neq 0$. Then we have

$$
\left\|n_{1} q_{4 k+1}^{\prime} \alpha\right\|=\left\|n_{2} q_{4 k+1}^{\prime} \beta\right\| \leq\left|n_{2}\right|\left\|q_{4 k+1}^{\prime} \beta\right\|<\frac{\left|n_{2}\right|}{q_{4 k+2}^{\prime}}<\frac{\left|n_{2}\right|}{a_{4 k+2}^{\prime} q_{4 k+1}^{\prime}} .
$$

Thus, there exists an integer $p$ satisfying

$$
\left|\alpha-\frac{p}{n_{1} q_{4 k+1}^{\prime}}\right|<\frac{\left|n_{2}\right|}{\left|n_{1}\right| a_{4 k+2}^{\prime}\left(q_{4 k+1}^{\prime}\right)^{2}} .
$$

Choose $k$ large enough for $a_{4 k+2}^{\prime}>2\left|n_{1}\right|\left|n_{2}\right|$ to hold. Then, by Legendre's theorem (see e.g. Bug04, Theorem 1.8]), one gets $\frac{p}{n_{1} q_{4 k+1}^{\prime}}=\frac{p_{s}}{q_{s}}$ for some positive integer $s$. Since $q_{4 k+2}=q_{4 k+2}^{\prime}+1>a_{4 k+2}^{\prime} q_{4 k+1}^{\prime}>\left|n_{1}\right| q_{4 k+1}^{\prime}$, we get $s \leq 4 k+1$. Also from $q_{4 k+1}^{\prime}=q_{4 k+1}+1$, we get $s \neq 4 k+1$. If we assume $s \leq 4 k$, then

$$
\left|\alpha-\frac{p_{s}}{q_{s}}\right| \geq\left|\alpha-\frac{p_{4 k}}{q_{4 k}}\right| \geq \frac{1}{2 q_{4 k} q_{4 k+1}}>\frac{1}{2\left(q_{4 k+1}^{\prime}\right)^{2}}>\frac{\left|n_{1}\right|\left|n_{2}\right|}{a_{4 k+2}^{\prime}\left(q_{4 k+1}^{\prime}\right)^{2}},
$$

which is a contradiction to (4.1).

Organization of the proof. We will work mainly with the points of the first level provided by $E_{q_{4 k+1}, q_{4 k+1}^{\prime}}(\alpha, \beta)$. This will be sufficient to derive infinitely many lengths for the points in $E_{N}$, with $N=q_{4 k+1}=q_{4 k+1}^{\prime}-1$.

The study of the first level will be divided into Lemma 4.1 and Proposition 4.2. The main difficulty here is that the map $\phi$ of Lemma 2.1 provides points that can be located either on the right, or on the left of a point (Assumption (2.3) does not hold).

Distribution of the points of $E_{q_{4 k+1}, q_{4 k+1}^{\prime}}(\alpha, \beta)$. We now consider points of the first level provided by $E_{q_{4 k+1}, q_{4 k+1}^{\prime}}(\alpha, \beta)$. Recall that $q_{4 k+1}^{\prime}=q_{4 k+1}+1$. With the notation of Lemma 2.1, put $i=j=4 k+1$. Observe that $b^{\prime}=1$. We consider

$$
\delta_{k}:=\left\|q_{4 k-1}^{\prime} \beta\right\|-\left\|q_{4 k} \alpha\right\| .
$$

According to Lemma 4.1 below, one has $\delta_{k}>0$.

Note that there are more than the 4 lengths of Lemma 2.1 since Assumption (2.3) is not satisfied. Indeed, one has $-\left\|q_{4 k} \alpha\right\|+\left\|q_{4 k-1}^{\prime} \beta\right\|=\delta_{k}>0$, which contradicts $\left\|q_{4 k+1}^{\prime} \beta\right\|<\left\|q_{4 k} \alpha\right\|-\left\|q_{4 k}^{\prime} \beta\right\|$, by noticing that $\left\|q_{4 k-1}^{\prime} \beta\right\|=$ $\left\|q_{4 k}^{\prime} \beta\right\|+\left\|q_{4 k+1}^{\prime} \beta\right\|$, since $a_{4 k+1}^{\prime}=1$. However, even if Assumption (2.3) is not satisfied, Lemma 2.1 provides a convenient expression $\phi$ for the neighbor map, that will be used in the proof of Proposition 4.2 below, showing that there are at most 12 lengths. 
Lemma 4.1. One has, for all $k$ :

$$
0<2 \delta_{k} a_{4 k+1}<\left\|q_{4 k+1} \alpha\right\|<\left\|q_{4 k+1}^{\prime} \beta\right\| .
$$

Proof. We have

$$
\left\|q_{k-1} \alpha\right\|=\frac{1}{q_{k}+q_{k-1} \frac{\left\|q_{k} \alpha\right\|}{\| q_{k-1} \alpha}}=\frac{1}{q_{k}+\frac{q_{k-1}}{a_{k+1}+\frac{1}{a_{k+2}+\ddots}} .}
$$

Hence we get

$$
\begin{aligned}
\left\|q_{4 k} \alpha\right\| & =\frac{1}{R_{k+1}-1+\frac{4 Q_{k}+3 R_{k}+1}{6 Q_{k}+4 R_{k}+s}}=\frac{1}{R_{k+1}-\frac{1}{3}+\frac{R_{k}+3-2 s}{3\left(6 Q_{k}+4 R_{k}+s\right)}} \\
\left\|q_{4 k-1}^{\prime} \beta\right\| & =\frac{1}{R_{k+1}-2 Q_{k}-R_{k}+\frac{2 Q_{k}+R_{k}}{1+\frac{1}{6 Q_{k}+4 R_{k}-1+s^{\prime}}}}=\frac{1}{R_{k+1}-\frac{1}{3}+\frac{R_{k}+s^{\prime}}{3\left(6 Q_{k}+4 R_{k}+s^{\prime}\right)}}
\end{aligned}
$$

where

$$
s:=\frac{1}{a_{4 k+3}+\frac{1}{a_{4 k+4}+\cdot}}, \quad s^{\prime}:=\frac{1}{a_{4 k+3}^{\prime}+\frac{1}{a_{4 k+4}^{\prime}+\cdot}}
$$

satisfying that

$$
\begin{aligned}
& \frac{6 Q_{k+1}+3 R_{k+1}+1}{8 Q_{k+1}+4 R_{k+1}+1}=\frac{3 a_{4 k+5}+4}{4 a_{4 k+5}+5}<s<\frac{3 a_{4 k+5}+1}{4 a_{4 k+5}+1}=\frac{6 Q_{k+1}+3 R_{k+1}-2}{8 Q_{k+1}+4 R_{k+1}-3} \\
& \frac{4 Q_{k+1}+3 R_{k+1}-2}{8 Q_{k+1}+6 R_{k+1}-3}=\frac{a_{4 k+4}^{\prime}}{2 a_{4 k+4}^{\prime}+1}<s^{\prime}<\frac{a_{4 k+4}^{\prime}+1}{2 a_{4 k+4}^{\prime}+3}=\frac{4 Q_{k+1}+3 R_{k+1}-1}{8 Q_{k+1}+6 R_{k+1}-1} .
\end{aligned}
$$

Then, we have

$$
\delta_{k}=\frac{\frac{R_{k}+3-2 s}{3\left(6 Q_{k}+4 R_{k}+s\right)}-\frac{R_{k}+s^{\prime}}{3\left(6 Q_{k}+4 R_{k}+s^{\prime}\right)}}{\left(R_{k+1}-\frac{1}{3}+\frac{R_{k}+s^{\prime}}{3\left(6 Q_{k}+4 R_{k}+s^{\prime}\right)}\right)\left(R_{k+1}-\frac{1}{3}+\frac{R_{k}+3-2 s}{3\left(6 Q_{k}+4 R_{k}+s\right)}\right)} .
$$

By elementary computation we get

$$
\frac{1}{3\left(6 Q_{k}+5 R_{k}\right) R_{k+1}^{2}}<\delta_{k}<\frac{1}{3\left(6 Q_{k}+4 R_{k}\right)\left(R_{k+1}-\frac{1}{3}\right)^{2}} .
$$

Also we have

$$
\left\|q_{4 k+1} \alpha\right\|=\frac{1}{Q_{k+1}+1+\left(R_{k+1}-1\right) s}, \quad\left\|q_{4 k+1}^{\prime} \beta\right\|=\frac{1}{Q_{k+1}+R_{k+1} s^{\prime}},
$$

thus

$$
\begin{aligned}
0<2 \delta_{k} a_{4 k+1}<\frac{1}{3\left(R_{k+1}-\frac{1}{3}\right)^{2}} & <\frac{1}{Q_{k+1}+R_{k+1}} \\
& <\left\|q_{4 k+1} \alpha\right\|<\left\|q_{4 k+1}^{\prime} \beta\right\|<\frac{1}{Q_{k+1}} .
\end{aligned}
$$


Proposition 4.2. Let $\alpha, \beta$ given by the construction above. We consider points of the first level provided by $E_{q_{4 k+1}, q_{4 k+1}^{\prime}}(\alpha, \beta)$. The neighbor map $\Phi=$ $\Phi_{q_{4 k+1}, q_{4 k+1}^{\prime}}$ satisfies the following.

(1) If $q_{4 k} \leq n<q_{4 k+1}, q_{4 k-1}^{\prime} \leq m<q_{4 k+1}^{\prime}$, then

$\Phi(n \alpha+m \beta)=\left(n-q_{4 k}\right) \alpha+\left(m-q_{4 k-1}^{\prime}\right) \beta, \quad$ and $\quad \Delta(n, m)=\delta_{k}$.

(2) If $0 \leq n<q_{4 k-1}, q_{4 k+1}^{\prime}-a_{4 k+1} q_{4 k-1}^{\prime} \leq m<q_{4 k+1}^{\prime}$, then

$$
\begin{aligned}
\Phi(n \alpha+m \beta) & =\left(n+a_{4 k+1} q_{4 k}\right) \alpha+\left(m+a_{4 k+1} q_{4 k-1}^{\prime}-q_{4 k+1}^{\prime}\right) \beta, \\
\Delta(n, m) & =\left\|q_{4 k+1}^{\prime} \beta\right\|-a_{4 k+1} \delta_{k} .
\end{aligned}
$$

(3) If $q_{4 k-1} \leq n<q_{4 k}, q_{4 k+1}^{\prime}-\left(a_{4 k+1}-1\right) q_{4 k-1}^{\prime} \leq m<q_{4 k+1}^{\prime}$, then

$$
\begin{aligned}
\Phi(n \alpha+m \beta) & =\left(n+\left(a_{4 k+1}-1\right) q_{4 k}\right) \alpha+\left(m+\left(a_{4 k+1}-1\right) q_{4 k-1}^{\prime}-q_{4 k+1}^{\prime}\right) \beta, \\
\Delta(n, m) & =\left\|q_{4 k+1}^{\prime} \beta\right\|-\left(a_{4 k+1}-1\right) \delta_{k} .
\end{aligned}
$$

(4) If $q_{4 k-1} \leq n<2 q_{4 k-1}, q_{4 k+1}^{\prime}-a_{4 k+1} q_{4 k-1}^{\prime} \leq m<q_{4 k+1}^{\prime}-\left(a_{4 k+1}-\right.$ 1) $q_{4 k-1}^{\prime}$, then

$$
\begin{aligned}
\Phi(n \alpha+m \beta) & =\left(n+2 a_{4 k+1} q_{4 k}-q_{4 k+1}\right) \alpha+\left(m+2 a_{4 k+1} q_{4 k-1}^{\prime}-q_{4 k+1}^{\prime}\right) \beta, \\
\Delta(n, m) & =\left\|q_{4 k+1} \alpha\right\|+\left\|q_{4 k+1}^{\prime} \beta\right\|-2 a_{4 k+1} \delta_{k} .
\end{aligned}
$$

(5) If $2 q_{4 k-1} \leq n<q_{4 k}, q_{4 k+1}^{\prime}-a_{4 k+1} q_{4 k-1}^{\prime} \leq m<q_{4 k+1}^{\prime}-\left(a_{4 k+1}-\right.$ 1) $q_{4 k-1}^{\prime}$, then

$$
\begin{aligned}
\Phi(n \alpha+m \beta)= & \left(n+\left(2 a_{4 k+1}-1\right) q_{4 k}-q_{4 k+1}\right) \alpha \\
& +\left(m+\left(2 a_{4 k+1}-1\right) q_{4 k-1}^{\prime}-q_{4 k+1}^{\prime}\right) \beta, \\
\Delta(n, m)= & \left\|q_{4 k+1} \alpha\right\|+\left\|q_{4 k+1}^{\prime} \beta\right\|-\left(2 a_{4 k+1}-1\right) \delta_{k} .
\end{aligned}
$$

(6) If $0 \leq n<q_{4 k-1}, q_{4 k+1}^{\prime}-\left(a_{4 k+1}+1\right) q_{4 k-1}^{\prime} \leq m<q_{4 k+1}^{\prime}-a_{4 k+1} q_{4 k-1}^{\prime}$, then

$$
\begin{aligned}
\Phi(n \alpha+m \beta) & =\left(n+2 a_{4 k+1} q_{4 k}-q_{4 k+1}\right) \alpha+\left(m+2 a_{4 k+1} q_{4 k-1}^{\prime}-q_{4 k+1}^{\prime}\right) \beta, \\
\Delta(n, m) & =\left\|q_{4 k+1} \alpha\right\|+\left\|q_{4 k+1}^{\prime} \beta\right\|-2 a_{4 k+1} \delta_{k} .
\end{aligned}
$$

(7) If $q_{4 k-1} \leq n<q_{4 k}, q_{4 k+1}^{\prime}-\left(a_{4 k+1}+1\right) q_{4 k-1}^{\prime} \leq m<q_{4 k+1}^{\prime}-a_{4 k+1} q_{4 k-1}^{\prime}$, then

$$
\begin{aligned}
\Phi(n \alpha+m \beta) & =\left(n+a_{4 k+1} q_{4 k}-q_{4 k+1}\right) \alpha+\left(m+a_{4 k+1} q_{4 k-1}^{\prime}\right) \beta, \\
\Delta(n, m) & =\left\|q_{4 k+1} \alpha\right\|-a_{4 k+1} \delta_{k} .
\end{aligned}
$$

(8) If $0 \leq n<q_{4 k}, q_{4 k+1}^{\prime}-\left(a_{4 k+1}+c+1\right) q_{4 k-1}^{\prime} \leq m<q_{4 k+1}^{\prime}-\left(a_{4 k+1}+\right.$ c) $q_{4 k-1}^{\prime}$, where $1 \leq c \leq a_{4 k+1}-1$, then

$$
\begin{aligned}
\Phi(n \alpha+m \beta) & =\left(n+\left(a_{4 k+1}+c\right) q_{4 k}-q_{4 k+1}\right) \alpha+\left(m+\left(a_{4 k+1}+c\right) q_{4 k-1}^{\prime}\right) \beta, \\
\Delta(n, m) & =\left\|q_{4 k+1} \alpha\right\|-\left(a_{4 k+1}+c\right) \delta_{k} .
\end{aligned}
$$


(9) If $0 \leq n<2 q_{4 k-1}, 0 \leq m<q_{4 k+1}^{\prime}-2 a_{4 k+1} q_{4 k-1}^{\prime}$, then

$$
\begin{gathered}
\Phi(n \alpha+m \beta)=\left(n+2 a_{4 k+1} q_{4 k}-q_{4 k+1}\right) \alpha+\left(m+2 a_{4 k+1} q_{4 k-1}^{\prime}\right) \beta, \\
\Delta(n, m)=\left\|q_{4 k+1} \alpha\right\|-2 a_{4 k+1} \delta_{k} . \\
\text { (10) If } 2 q_{4 k-1} \leq n<q_{4 k}, 0 \leq m<q_{4 k+1}^{\prime}-\left(2 a_{4 k+1}-1\right) q_{4 k-1}^{\prime}, \text { then } \\
\Phi(n \alpha+m \beta)=\left(n+\left(2 a_{4 k+1}-1\right) q_{4 k}-q_{4 k+1}\right) \alpha+\left(m+\left(2 a_{4 k+1}-1\right) q_{4 k-1}^{\prime}\right) \beta, \\
\Delta(n, m)=\left\|q_{4 k+1} \alpha\right\|-\left(2 a_{4 k+1}-1\right) \delta_{k} . \\
\text { (11) If }(c-1) q_{4 k}+2 q_{4 k-1} \leq n<c q_{4 k}+2 q_{4 k-1}, 0 \leq m<q_{4 k-1}^{\prime}, \text { where } \\
1 \leq c \leq a_{4 k+1}-1, \text { then } \\
\Phi(n \alpha+m \beta)=\left(n+\left(2 a_{4 k+1}-c\right) q_{4 k}-q_{4 k+1}\right) \alpha+\left(m+\left(2 a_{4 k+1}-c\right) q_{4 k-1}^{\prime}\right) \beta, \\
\Delta(n, m)=\left\|q_{4 k+1} \alpha\right\|-\left(2 a_{4 k+1}-c\right) \delta_{k} . \\
\text { (12) If }\left(a_{4 k+1}-1\right) q_{4 k}+2 q_{4 k-1} \leq n<q_{4 k+1}, 0 \leq m<q_{4 k-1}^{\prime}, \text { then } \\
\Phi(n \alpha+m \beta)=\left(n+a_{4 k+1} q_{4 k}-q_{4 k+1}\right) \alpha+\left(m+a_{4 k+1} q_{4 k-1}^{\prime}\right) \beta, \\
\Delta(n, m)=\left\|q_{4 k+1} \alpha\right\|-a_{4 k+1} \delta_{k} .
\end{gathered}
$$

Remark 4.3. Observe that there are overlapped regions between points corresponding to Case (8) and Case (10) (when $c=a_{4 k+1}-1$ ), and between Case (10) and Case (11) (when $c=1$ ).

Proof. Let $\bar{\Phi}$ and $\bar{\Delta}$ stand for the functions defined in the statement of the proposition. We want to prove that $\bar{\Phi}$ coincides with $\Phi$ on $E_{q_{4 k+1}, q_{4 k+1}^{\prime}}$, and that similarly $\bar{\Delta}$ coincides with $\Delta$. The proof works as for Lemma 2.1; we will show that the sum of the lengths provided by $\bar{\Delta}$ (with multiplicities) equals 1 .

According to Lemma 4.1, one checks that the lengths $\bar{\Delta}$ are all nonnegative. The intervals for the pairs $(n, m)$ in Proposition 4.2 are also welldefined. Indeed, one checks that $q_{4 k}-2 q_{4 k-1}>0$, by noticing that $a_{4 k}=3$, and also $q_{4 k+1}^{\prime}-\left(2 a_{4 k+1}+1\right) q_{4 k-1}^{\prime}>0$.

One has $q_{4 k+1}^{\prime}=q_{4 k+1}+1$. With the notation of Lemma 2.1, one has $b^{\prime}=1$. Since $q_{4 k-1}^{\prime}=q_{4 k+1}^{\prime}-a_{4 k+1}^{\prime} q_{4 k}^{\prime}=q_{4 k+1}^{\prime}-q_{4 k}^{\prime}$, we have

$$
\left|m-q_{4 k}^{\prime}\right|_{q_{4 k+1}^{\prime}}=\left|m+q_{4 k-1}^{\prime}\right|_{q_{4 k+1}^{\prime}} .
$$

As in Lemma 2.1, we consider the cyclic permutation $\phi$ on $E_{q_{4 k+1}, q_{4 k+1}^{\prime}}(\alpha, \beta)$ defined, for all $(n, m) \in \mathcal{E}_{q_{4 k+1}, q_{4 k+1}^{\prime}}$, as:

$$
\begin{aligned}
\phi(n \alpha+m \beta) & =\left|n+q_{4 k}\right|_{q_{4 k+1}} \alpha+\left|m-q_{4 k}^{\prime}\right|_{q_{4 k+1}^{\prime}} \beta \\
& =\left|n+q_{4 k}\right|_{q_{4 k+1}} \alpha+\left|m+q_{4 k-1}^{\prime}\right|_{q_{4 k+1}^{\prime}} \beta .
\end{aligned}
$$




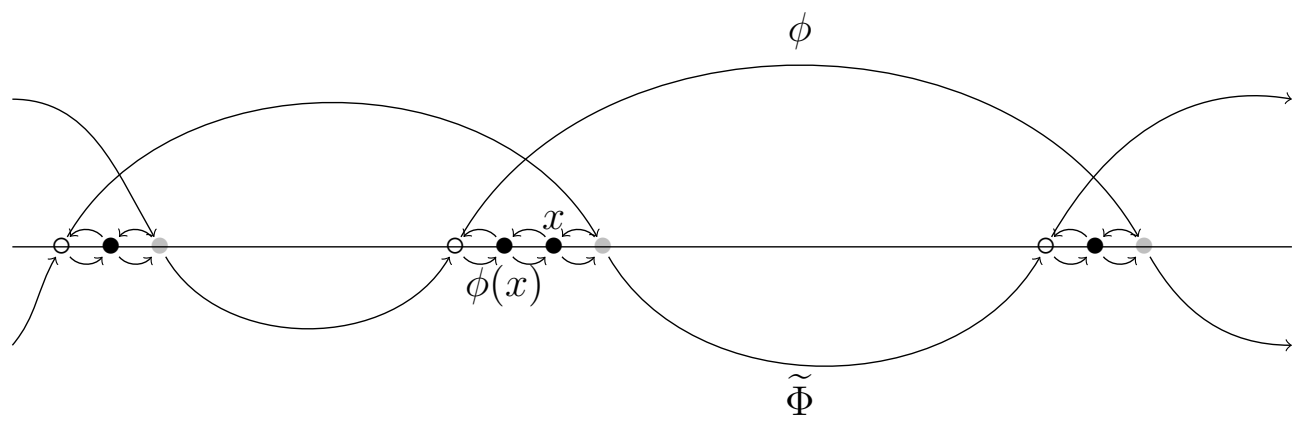

Figure 6 . The points marked by o are elements of $G$. Elements of $\phi(G)$ are marked in light gray.

We first provide some dynamical insight on the way the 12 lengths in Proposition 4.2 have been obtained. As stressed before, Assumption (2.3) is not satisfied, and the neighbor map $\Phi$ is not equal to $\phi$. In fact, there are points $x$ for which $\phi(x)$ is obtained from $x$ by performing a clockwise jump of $\delta_{k}$, but there are also points $x$ for which $\phi(x)$ is located in the anticlockwise direction $(\phi(x)<x)$, with $x$ being the clockwise neighbor of $\phi(x)$. However, the map $\Phi$ can ce be recovered by performing suitable inductions of the map $\phi$ on the set of points for which $\phi(x)>x$. Let

$G:=\left\{n \alpha+m \beta: q_{4 k+1}-q_{4 k} \leq n<q_{4 k+1}\right.$ or $\left.q_{4 k+1}^{\prime}-q_{4 k-1}^{\prime}=q_{4 k}^{\prime} \leq m<q_{4 k+1}^{\prime}\right\}$.

One has $G \subset E_{q_{4 k+1}, q_{4 k+1}^{\prime}}(\alpha, \beta)$. Then $G$ is the set of points such that $\phi(x)>$ $x$, that is, $\phi(x)$ is obtained from $x$ by performing a clockwise jump of $\delta_{k}=$ $\left\|q_{4 k-1}^{\prime} \beta\right\|-\left\|q_{4 k} \alpha\right\|>0$. Elements $n \alpha+m \beta$ in $\phi(G)$ are such that $0 \leq n<q_{4 k}$, or $0 \leq m<q_{4 k-1}^{\prime}$. This is the complement of the set of $(n, m)$ corresponding to Case (1). Let $F_{G}$ be defined on $E_{q_{4 k+1}, q_{4 k+1}^{\prime}}(\alpha, \beta)$ as the first entering time of $\phi$ to $G$, that is,

$$
F_{G}(n \alpha+m \beta):=\min \left\{\ell \geq 0: \phi^{\ell}(n \alpha+m \beta) \in G\right\} .
$$

Also, define $S_{G}$ as the second entering time of $\phi$ to $G$ :

$$
\begin{aligned}
S_{G}(n \alpha+m \beta): & =\min \left\{\ell \geq F_{G}(n \alpha+m \beta)+1: \phi^{\ell}(n \alpha+m \beta) \in G\right\} \\
& =F_{G}\left(\phi^{F_{G}(n \alpha+m \beta)+1}(n \alpha+m \beta)\right)+F_{G}(n \alpha+m \beta) .
\end{aligned}
$$

We need to consider the second entering time to recover an element located in the clockwise direction.

Let us now define a map $\widetilde{\Phi}$ on $E_{q_{4 k+1}, q_{4 k+1}^{\prime}}(\alpha, \beta)$ as follows (see Figure 6 ):

$$
\widetilde{\Phi}(n \alpha+m \beta):= \begin{cases}\phi^{-1}(n \alpha+m \beta) & \text { if } n \alpha+m \beta \notin \phi(G), \\ \phi^{S_{G}(n \alpha+m \beta)}(n \alpha+m \beta) & \text { if } n \alpha+m \beta \in \phi(G) .\end{cases}
$$



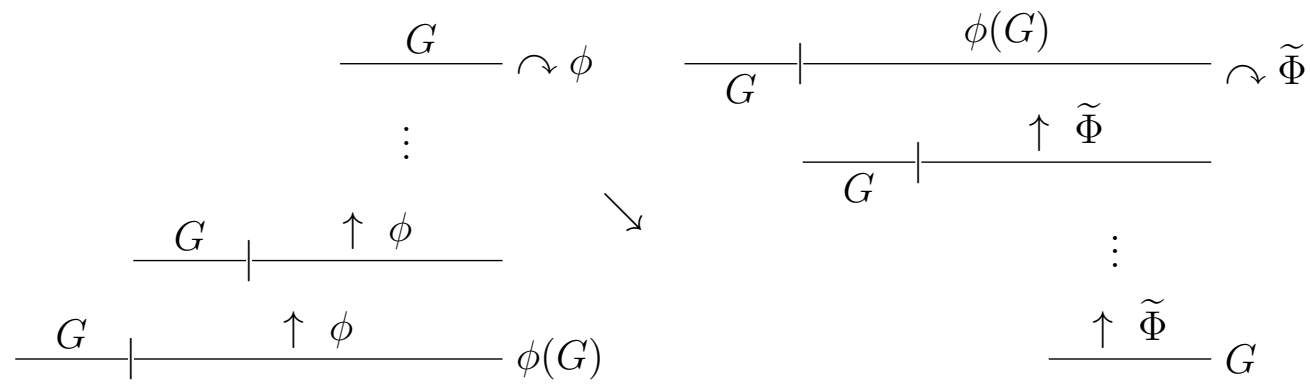

FiguRE 7. Each $k$-level of the tower is moved to the level of index $-k$, with the indices of tower on the left being positive, and negative on the right. The actions on the rooftops are $\phi$ and $\widetilde{\Phi}$, respectively.

The map $\widetilde{\Phi}$ is a cyclic permutation on $E_{q_{4 k+1}, q_{4 k+1}^{\prime}}(\alpha, \beta)$. This is illustrated by the skyscraper tower construction of Figure 7 (see for instance [Pet89, Page 40]). One can check that $\widetilde{\Phi}$ coincides with the function $\bar{\Phi}$ on $E_{q_{4 k+1}, q_{4 k+1}^{\prime}}(\alpha, \beta)$. We will not use this fact in the proof but, as said before, it aims at providing some insight on the organisation of the cases that occur in the statement of Proposition 4.2.

We now come back to the proof of Proposition 4.2. Let us count the number of points $(n, m)$ taking the same value $\bar{\Delta}$. There are

(a) $\left(q_{4 k+1}-q_{4 k}\right) q_{4 k}^{\prime}$ points such that $\bar{\Delta}(n, m)=\delta_{k}($ Case $(1))$;

(b) $q_{4 k-1} a_{4 k+1} q_{4 k-1}^{\prime}$ points such that $\bar{\Delta}(n, m)=\left\|q_{4 k+1}^{\prime} \beta\right\|-a_{4 k+1} \delta_{k}$ (Case $(2))$;

(c) $\left(q_{4 k}-q_{4 k-1}\right)\left(a_{4 k+1}-1\right) q_{4 k-1}^{\prime}$ points such that $\bar{\Delta}(n, m)=\left\|q_{4 k+1}^{\prime} \beta\right\|-$ $\left(a_{4 k+1}-1\right) \delta_{k}$ (Case $\left.(3)\right)$;

(d) $2 q_{4 k-1} q_{4 k-1}^{\prime}$ points such that $\bar{\Delta}(n, m)=\left\|q_{4 k+1} \alpha\right\|+\left\|q_{4 k+1}^{\prime} \beta\right\|-2 a_{4 k+1} \delta_{k}$ (Case (4) and (6));

(e) $\left(q_{4 k}-2 q_{4 k-1}\right) q_{4 k-1}^{\prime}$ points such that $\bar{\Delta}(n, m)=\left\|q_{4 k+1} \alpha\right\|+\left\|q_{4 k+1}^{\prime} \beta\right\|-$ $\left(2 a_{4 k+1}-1\right) \delta_{k}($ Case $(5))$;

(f) $2\left(q_{4 k}-q_{4 k-1}\right) q_{4 k-1}^{\prime}$ points such that $\bar{\Delta}(n, m)=\left\|q_{4 k+1} \alpha\right\|-a_{4 k+1} \delta_{k}$ (Case (7) and (12));

(g) $2 q_{4 k} q_{4 k-1}^{\prime}$ points such that $\bar{\Delta}(n, m)=\left\|q_{4 k+1} \alpha\right\|-\left(a_{4 k+1}+c\right) \delta_{k}$ for $1 \leq c \leq a_{4 k+1}-1$ (Case (8) and (11));

(h) another $\left(q_{4 k}-2 q_{4 k-1}\right)\left(q_{4 k+1}^{\prime}-\left(2 a_{4 k+1}+1\right) q_{4 k-1}^{\prime}\right)$ points such that $\bar{\Delta}(n, m)=\left\|q_{4 k+1} \alpha\right\|-\left(2 a_{4 k+1}-1\right) \delta_{k}$ (those points correspond to Case (10), Case (8), with $c=a_{4 k+1}-1$, but we do not take into account Case (11), with $c=1)$;

(i) $2 q_{4 k-1}\left(q_{4 k+1}^{\prime}-2 a_{4 k+1} q_{4 k-1}^{\prime}\right)$ points such that $\bar{\Delta}(n, m)=\left\|q_{4 k+1} \alpha\right\|-$ $2 a_{4 k+1} \delta_{k}$ (Case $\left.(9)\right)$. 
As noticed in Remark 4.3, there are overlaps between Case (8) and (10) $\left(c=a_{4 k+1}-1\right)$ and between Case (10) and (11) $(c=1)$. There are $\left(q_{4 k}-\right.$ $\left.2 q_{4 k-1}\right) q_{4 k-1}^{\prime}$ points in both intersections, thus in (h) the total number of points is

$$
\begin{aligned}
\left(q_{4 k}-2 q_{4 k-1}\right)\left(q_{4 k+1}^{\prime}-\left(2 a_{4 k+1}-1\right) q_{4 k-1}^{\prime}\right)-2\left(q_{4 k}-2 q_{4 k-1}\right) q_{4 k-1}^{\prime} & \\
= & \left(q_{4 k}-2 q_{4 k-1}\right)\left(q_{4 k+1}^{\prime}-\left(2 a_{4 k+1}+1\right) q_{4 k-1}^{\prime}\right) .
\end{aligned}
$$

We denote the sum of all the lengths $\bar{\Delta}$ of the intervals given in the statement of the proposition as

$$
S:=\sum_{0 \leq n \leq q_{4 k+1}, 0 \leq m<q_{4 k+1}^{\prime}} \bar{\Delta}(n, m)=S_{0}+S_{1}+S_{2}+S_{3},
$$

where $S_{0}$ corresponds to Case (a), $S_{1}$ to Case (b) and (c), $S_{2}$ to Case (d) and (e), and $S_{3}$ to the other cases. This yields

$$
\begin{aligned}
S_{0}:= & \left(q_{4 k+1}-q_{4 k}\right) q_{4 k}^{\prime} \delta_{k}, \\
S_{1}:= & q_{4 k-1} a_{4 k+1} q_{4 k-1}^{\prime}\left(\left\|q_{4 k+1}^{\prime} \beta\right\|-a_{4 k+1} \delta_{k}\right) \\
& +\left(q_{4 k}-q_{4 k-1}\right)\left(a_{4 k+1}-1\right) q_{4 k-1}^{\prime}\left(\left\|q_{4 k+1}^{\prime} \beta\right\|-\left(a_{4 k+1}-1\right) \delta_{k}\right), \\
S_{2}:= & 2 q_{4 k-1} q_{4 k-1}^{\prime}\left(\left\|q_{4 k+1} \alpha\right\|+\left\|q_{4 k+1}^{\prime} \beta\right\|-2 a_{4 k+1} \delta_{k}\right) \\
& +\left(q_{4 k}-2 q_{4 k-1}\right) q_{4 k-1}^{\prime}\left(\left\|q_{4 k+1} \alpha\right\|+\left\|q_{4 k+1}^{\prime} \beta\right\|-\left(2 a_{4 k+1}-1\right) \delta_{k}\right) .
\end{aligned}
$$

Let us prove that $S=1$. Since the sum of the lengths $\bar{\Delta}$ for Case (g) is

$$
\begin{aligned}
& 2 q_{4 k} q_{4 k-1}^{\prime} \sum_{c=1}^{a_{4 k+1}-1}\left(\left\|q_{4 k+1} \alpha\right\|-\left(a_{4 k+1}+c\right) \delta_{k}\right) \\
& =2 q_{4 k} q_{4 k-1}^{\prime}\left(a_{4 k+1}-1\right)\left(\left\|q_{4 k+1} \alpha\right\|-a_{4 k+1} \delta_{k}\right)-2 q_{4 k} q_{4 k-1}^{\prime} \frac{\left(a_{4 k+1}-1\right) a_{4 k+1} \delta_{k}}{2} \\
& =2 q_{4 k} q_{4 k-1}^{\prime}\left(a_{4 k+1}-1\right)\left\|q_{4 k+1} \alpha\right\|-3 q_{4 k} q_{4 k-1}^{\prime}\left(a_{4 k+1}-1\right) a_{4 k+1} \delta_{k},
\end{aligned}
$$

we get

$$
\begin{aligned}
S_{3}:= & 2\left(q_{4 k}-q_{4 k-1}\right) q_{4 k-1}^{\prime}\left(\left\|q_{4 k+1} \alpha\right\|-a_{4 k+1} \delta_{k}\right) \\
& +2 q_{4 k} q_{4 k-1}^{\prime}\left(a_{4 k+1}-1\right)\left\|q_{4 k+1} \alpha\right\|-3 q_{4 k} q_{4 k-1}^{\prime} a_{4 k+1}\left(a_{4 k+1}-1\right) \delta_{k} \\
& +\left(q_{4 k}-2 q_{4 k-1}\right)\left(q_{4 k+1}^{\prime}-\left(2 a_{4 k+1}+1\right) q_{4 k-1}^{\prime}\right)\left(\left\|q_{4 k+1} \alpha\right\|-\left(2 a_{4 k+1}-1\right) \delta_{k}\right) \\
& +2 q_{4 k-1}\left(q_{4 k+1}^{\prime}-2 a_{4 k+1} q_{4 k-1}^{\prime}\right)\left(\left\|q_{4 k+1} \alpha\right\|-2 a_{4 k+1} \delta_{k}\right) . \\
& 25
\end{aligned}
$$


Then we get

$$
\begin{aligned}
S_{1}= & \left(q_{4 k+1}-q_{4 k}\right) q_{4 k-1}^{\prime}\left\|q_{4 k+1}^{\prime} \beta\right\| \\
& -\left(\left(a_{4 k+1}-1\right)\left(q_{4 k+1}-q_{4 k}\right)+a_{4 k+1} q_{4 k-1}\right) q_{4 k-1}^{\prime} \delta_{k}, \\
S_{2}= & q_{4 k} q_{4 k-1}^{\prime}\left(\left\|q_{4 k+1} \alpha\right\|+\left\|q_{4 k+1}^{\prime} \beta\right\|\right)-\left(2 q_{4 k+1}-q_{4 k}\right) q_{4 k-1}^{\prime} \delta_{k}, \\
S_{3}= & q_{4 k}\left(q_{4 k+1}^{\prime}-q_{4 k-1}^{\prime}\right)\left\|q_{4 k+1} \alpha\right\| \\
& +\left(\left(a_{4 k+1}+1\right)\left(q_{4 k+1}+q_{4 k-1}\right)-q_{4 k}\right) q_{4 k-1}^{\prime} \delta_{k}-\left(2 q_{4 k+1}-q_{4 k}\right) q_{4 k+1}^{\prime} \delta_{k} .
\end{aligned}
$$

Therefore, we have

$$
\begin{aligned}
S= & q_{4 k} q_{4 k+1}^{\prime}\left\|q_{4 k+1} \alpha\right\|+q_{4 k+1} q_{4 k-1}^{\prime}\left\|q_{4 k+1}^{\prime} \beta\right\| \\
& +\left(q_{4 k+1}-q_{4 k}\right) q_{4 k}^{\prime} \delta_{k}+\left(q_{4 k+1}-q_{4 k}\right) q_{4 k-1}^{\prime} \delta_{k}-\left(2 q_{4 k+1}-q_{4 k}\right) q_{4 k+1}^{\prime} \delta_{k} \\
= & q_{4 k} q_{4 k+1}^{\prime}\left\|q_{4 k+1} \alpha\right\|+q_{4 k+1} q_{4 k-1}^{\prime}\left\|q_{4 k+1}^{\prime} \beta\right\|-q_{4 k+1} q_{4 k+1}^{\prime} \delta_{k} \\
= & q_{4 k} q_{4 k+1}^{\prime}\left\|q_{4 k+1} \alpha\right\|+q_{4 k+1} q_{4 k-1}^{\prime}\left\|q_{4 k+1}^{\prime} \beta\right\| \\
& -q_{4 k+1} q_{4 k+1}^{\prime}\left(\left\|q_{4 k}^{\prime} \beta\right\|+\left\|q_{4 k+1}^{\prime} \beta\right\|-\left\|q_{4 k} \alpha\right\|\right) \\
= & q_{4 k+1}^{\prime}\left(q_{4 k}\left\|q_{4 k+1} \alpha\right\|+q_{4 k+1}\left\|q_{4 k} \alpha\right\|\right)-q_{4 k+1}\left(q_{4 k}^{\prime}\left\|q_{4 k+1}^{\prime} \beta\right\|+q_{4 k+1}^{\prime}\left\|q_{4 k}^{\prime} \beta\right\|\right) \\
= & q_{4 k+1}^{\prime}-q_{4 k+1}=1 .
\end{aligned}
$$

Hence, the intervals $(n \alpha+m \beta, \bar{\Phi}(n \alpha+m \beta))$ never overlap (as intervals of $\mathbb{T})$, which implies that $\bar{\Phi}(n \alpha+m \beta)$ is the neighbor point of $n \alpha+m \beta$, that is, $\bar{\Phi}=\Phi$, which ends the proof of Proposition 4.2 .

End of the proof. According to Cases (11), (12) of Proposition 4.2, one has, for $n=c q_{4 k}+2 q_{4 k-1}, 0 \leq c \leq a_{4 k+1}-1$ and $m=0$ :

$$
\begin{aligned}
& \Phi_{q_{4 k+1}, q_{4 k+1}^{\prime}}\left(\left(c q_{4 k}+2 q_{4 k-1}\right) \alpha+0 \beta\right) \\
& \quad=\left(\left(2 a_{4 k+1}-1\right) q_{4 k}-q_{4 k+1}+2 q_{4 k-1}\right) \alpha+\left(2 a_{4 k+1}-c-1\right) q_{4 k-1}^{\prime} \beta \\
& \quad=\left(q_{4 k+1}-q_{4 k}\right) \alpha+\left(2 a_{4 k+1}-c-1\right) q_{4 k-1}^{\prime} \beta .
\end{aligned}
$$

Let $N=q_{4 k+1}=q_{4 k+1}^{\prime}-1$. For each $0 \leq c \leq a_{4 k+1}-1$, the following pair of points belongs to $E_{N}(\alpha, \beta)$ :

$$
\begin{aligned}
\left(c q_{4 k}+2 q_{4 k-1}\right) \alpha & \in E_{N}(\alpha, \beta), \\
\left(q_{4 k+1}-q_{4 k}\right) \alpha+\left(2 a_{4 k+1}-c-1\right) q_{4 k-1}^{\prime} \beta & \in E_{N}(\alpha, \beta) .
\end{aligned}
$$

Since $E_{N}(\alpha, \beta) \subset E_{q_{4 k+1}, q_{4 k+1}^{\prime}}(\alpha, \beta)$ and the pairs of points above are adjacent points of $E_{q_{4 k+1}, q_{4 k+1}^{\prime}}(\alpha, \beta)$, we have for each $0 \leq c \leq a_{4 k+1}-1$ :

$$
\begin{aligned}
& \left(q_{4 k+1}-q_{4 k}\right) \alpha+\left(2 a_{4 k+1}-c-1\right) q_{4 k-1}^{\prime} \beta-\left(c q_{4 k}+2 q_{4 k-1}\right) \alpha \\
& \quad=\left(\left(2 a_{4 k+1}-c-1\right) q_{4 k}-q_{4 k+1}\right) \alpha+\left(2 a_{4 k+1}-c-1\right) q_{4 k-1}^{\prime} \beta \\
& \quad=\left\|q_{4 k+1} \alpha\right\|-\left(2 a_{4 k+1}-c-1\right) \delta_{k} \in \Delta\left(E_{N}(\alpha, \beta)\right) .
\end{aligned}
$$


Since the sequence of partial quotients $\left(a_{4 k+1}\right)_{k}$ goes to infinity, we conclude that

$$
\limsup _{N \rightarrow+\infty} \# \Delta E_{N}(\alpha, \beta)=\infty,
$$

which completes the proof of Theorem 1.1 (ii).

\section{REFERENCES}

[AB98] Pascal Alessandri and Valérie Berthé, Three distance theorems and combinatorics on words, Enseign. Math. (2) 44 (1998), no. 1-2, 103-132. MR 1643286

[ADG $\left.{ }^{+} 16\right]$ Faustin Adiceam, David Damanik, Franz Gähler, Uwe Grimm, Alan Haynes, Antoine Julien, Andrés Navas, Lorenzo Sadun, and Barak Weiss, Open problems and conjectures related to the theory of mathematical quasicrystals, Arnold Math. J. 2 (2016), no. 4, 579-592. MR 3564887

$\left[\mathrm{BHJ}^{+}\right.$12] Pavel M. Bleher, Youkow Homma, Lyndon L. Ji, Roland K. W. Roeder, and Jeffrey D. Shen, Nearest neighbor distances on a circle: multidimensional case, J. Stat. Phys. 146 (2012), no. 2, 446-465. MR 2873022

[Ble91] P. M. Bleher, The energy level spacing for two harmonic oscillators with generic ratio of frequencies, J. Statist. Phys. 63 (1991), no. 1-2, 261-283. MR 1115584

[BS08] Ian Biringer and Benjamin Schmidt, The three gap theorem and Riemannian geometry, Geom. Dedicata 136 (2008), 175-190. MR 2443351

[BT02] Valérie Berthé and Robert Tijdeman, Balance properties of multi-dimensional words, Theoret. Comput. Sci. 273 (2002), no. 1-2, 197-224, WORDS (Rouen, 1999). MR 1872450

[Bug04] Yann Bugeaud, Approximation by algebraic numbers, Cambridge Tracts in Mathematics, vol. 160, Cambridge University Press, Cambridge, 2004. MR 2136100

[BV00] Valérie Berthé and Laurent Vuillon, Tilings and rotations on the torus: a two-dimensional generalization of Sturmian sequences, Discrete Math. 223 (2000), no. 1-3, 27-53. MR 1782038

[CG76] F. R. K. Chung and R. L. Graham, On the set of distances determined by the union of arithmetic progressions, Ars Combinatoria 1 (1976), no. 1, 57-76. MR 0412118

[CGVZ02] C. Cobeli, G. Groza, M. Vâjâitu, and A. Zaharescu, Generalization of a theorem of Steinhaus, Colloq. Math. 92 (2002), no. 2, 257-266. MR 1899442

[Che00] Nicolas Chevallier, Three distance theorem and grid graph, Discrete Math. 223 (2000), no. 1-3, 355-362. MR 1782060

[Che07]_, Cyclic groups and the three distance theorem, Canad. J. Math. 59 (2007), no. 3, 503-552.

[Che14] Stepped hyperplane and extension of the three distance theorem, Ergodic theory and dynamical systems, De Gruyter Proc. Math., De Gruyter, Berlin, 2014, pp. 81-92. MR 3220099

[Dia17] (Dia) Taha Diaaeldin, The three gaps theorems, interval exchange transformations, and zippered rectangles, arXiv:1708.04380, preprint, 2017.

[FH95] Aviezri S. Fraenkel and Ron Holzman, Gap problems for integer part and fractional part sequences, J. Number Theory 50 (1995), no. 1, 66-86. MR 1310736

[FS92] E. Fried and Vera T. Sós, A generalization of the three-distance theorem for groups, Algebra Universalis 29 (1992), no. 1, 136-149. MR 1145560

[GS93] J. F. Geelen and R. J. Simpson, A two-dimensional Steinhaus theorem, Australas. J. Combin. 8 (1993), 169-197.

[Hal65] John H. Halton, The distribution of the sequence $\{n \xi\}(n=0,1,2, \cdots)$, Proc. Cambridge Philos. Soc. 61 (1965), 665-670. MR 0202668 
[HJKW17] Alan Haynes, Antoine Julien, Henna Koivusalo, and James Walton, Statistics of patterns in typical cut and project sets, arXiv:1702.04041, preprint, 2017.

[HKWS16] Alan Haynes, Henna Koivusalo, James Walton, and Lorenzo Sadun, Gaps problems and frequencies of patches in cut and project sets, Math. Proc. Cambridge Philos. Soc. 161 (2016), no. 1, 65-85. MR 3505670

[HM17] A. Haynes and J. Marklof, Higher dimensional Steinhaus and Slater problems via homogeneous dynamics, arXiv:1707.04094, preprint, 2017.

[Lan91] M. Langevin, Stimulateur cardiaque et suites de Farey, Period. Math. Hungar. 23 (1991), no. 1, 75-86. MR 1141354

[Lan95] Serge Lang, Introduction to Diophantine approximations, second ed., Springer-Verlag, New York, 1995. MR 1348400

[Lia79] Frank M. Liang, A short proof of the $3 d$ distance theorem, Discrete Math. 28 (1979), no. 3, 325-326. MR 548632

[MS17] J. Marklof and A. Strömbergsson, The three gap theorem and the space of lattices, arXiv:1612.04906v2, to appear in the American Mathematical Monthly preprint, 2017.

[Pet89] Karl Petersen, Ergodic theory, Cambridge Studies in Advanced Mathematics, vol. 2, Cambridge University Press, Cambridge, 1989, Corrected reprint of the 1983 original. MR 1073173

[PSZ16] Geremí as Polanco, Daniel Schultz, and Alexandru Zaharescu, Continuous distributions arising from the three gap theorem, Int. J. Number Theory 12 (2016), no. 7, 1743-1764. MR 3544409

[S5́8] Vera T. Sós, On the distribution mod 1 of the sequence na., Ann. Univ. Sci. Budap. Rolando Eötvös, Sect. Math. 1 (1958), 127-134.

[Ś59] S. Świerczkowski, On successive settings of an arc on the circumference of a circle, Fund. Math. 46 (1959), 187-189. MR 0104651

[Sla64] N. B. Slater, Distribution problems and physical applications, Compositio Math. 16 (1964), 176-183 (1964). MR 0174917

[Sla67] Noel B. Slater, Gaps and steps for the sequence $n \theta \bmod 1$, Proc. Cambridge Philos. Soc. 63 (1967), 1115-1123. MR 0217019

[Suŕ58] J. Suŕanyi, Über die Anordnung der Vielfachen einer reellen Zahl mod 1., Ann. Univ. Sci. Budap. Rolando Eötvös, Sect. Math. 1 (1958), 107-111 (German).

[Vij08] Sujith Vijay, Eleven Euclidean distances are enough, J. Number Theory 128 (2008), no. 6, 1655-1661. MR 2419185

[vR88] Tony van Ravenstein, The three gap theorem (Steinhaus conjecture), J. Austral. Math. Soc. Ser. A 45 (1988), no. 3, 360-370. MR 957201

IRIF, CNRS UMR 8243, Université PARIS Diderot - Paris 7, CASE 7014, 75205 Paris Cedex 13, France

E-mail address: berthe@irif.fr

Department of Mathematics Education Dongguk University - Seoul 30 Pildong-Ro 1-Gil, Jung-Gu SeOul, 04620 Korea

E-mail address: kim2010@dongguk.edu 